\title{
Motion Control of Vehicles with Trailers Using Transverse Function Approach
}

\section{Controller Properties Analysis}

\author{
Dariusz Pazderski • Dorota K. Waśkowicz • \\ Krzysztof Kozłowski
}

Received: 2 February 2013 / Accepted: 30 May 2013 / Published online: 2 October 2013

(C) The Author(s) 2013. This article is published with open access at Springerlink.com

\begin{abstract}
This paper is focused on analysis of the control solution using the transverse function approach. The controller considered here is designed for a nonholonomic vehicle with on-axle passive trailers. The main problem investigated is the optimal parametrization of the transverse functions in order to ensure low sensitivity to the measurement noise and high tracking accuracy. Theoretical analysis referring to transverse function scaling using dilation is illustrated by results of extensive numerical simulations. Taking into account these results the controller properties are considered. Finally, possibility of the controller implementation is discussed.
\end{abstract}

Keywords Nonholonomic multi-body vehicles • Transverse functions - Chained systems . Controller implementation $\cdot$ Robustness to the measurement noise

D. Pazderski $(\bowtie) \cdot$ D. K. Waśkowicz · K. Kozłowski Chair of Control and Systems Engineering, Poznan University of Technology, ul. Piotrowo 3a, 60-965 Poznan, Poland e-mail: dariusz.pazderski@put.poznan.pl

D. K. Waśkowicz

e-mail: dorota.waskowicz@gmail.com

K. Kozłowski

e-mail: krzysztof.kozlowski@put.poznan.pl

\section{Introduction}

Motion control of nonholonomic systems has become of great importance for many applications in robotics. Theoretical issues concerning control of such systems were formulated in the 70's and 80's of the twentieth century. As a result, it was found that the most difficult control task defined for such a class of systems is stabilization at the point using a closed-loop feedback. In particular, the well-known Brockett's theorem implies that there does not exist any classical time invariant smooth static state feedback which solves the regulation problem for these systems.

Consequently, many open-loop strategies were developed $[3,5,7,18]$ and alternative feedbacks were proposed. It was shown that time-varying signals which introduce permanent excitation to the closed-loop system give possibility to establish asymptotic stability [23, 26]. Another closed-loop control approach takes advantage of feedback singularity at the desired point $[2,10,27]$. Hence, it guarantees a solution to the convergence problem but no stability defined by Lyapunov theory. It is known that smooth time varying asymptotic stabilizers and discontinuous controllers have opposite properties. The former are quite robust to unknown internal or external disturbances but the convergence rate is typically weak. The latter are extremely sensitive at the desired point as a result of discontinuity but the convergence is usually 
fast. This issue have been reported in some papers $[6,9,22,24,25]$.

Besides the point stabilization, the tracking control for nonholonomic systems is also an important issue. Although, in some particular cases a relatively simple feedback based on linear techniques can be developed (cf. [14]), the general control solution is quite hard to establish. It turns out that it is a challenge to solve tracking task assuming that the reference trajectory does not introduce persistence excitation to the closed-loop control system or is non admissible (namely it can violates the phase constraints).

One of the latest method of stabilization have been formulated by Morin and Samson [11]. It takes advantage of transverse functions and refers to fundamental properties of an affine control system and its own control Lie algebra. The most important feature of this approach is a relaxation of the fundamental control task. Namely, it is assumed that trajectory error converges to the invariant set which corresponds to the well-defined neighborhood of the desired point. It can be stated that the transverse functions define some kind of trajectory in the configuration space such that its derivative along with vector fields of the control system span the tangent space. The time-evolution of the transverse functions are governed by an augmented dynamics that is dependent on the tracking error. This approach has been effectively used to control invariant systems (defined on Lie group) for which global stabilization result can be guaranteed. It is a universal approach that ensures practical (in some cases asymptotic) stabilization with respect to the regulation and trajectory tracking problems. Recently, the controller based on the transverse functions have been adapted by Morin and Samson to some class of non invariant systems including a car-like kinematics and a unicycle with N-on-axle trailers [16, 17].

So far not many papers address implementation and practical issues of this control method. In fact only a few results have been reported with respect to relatively low dimensional systems including two-wheeled and skid-steering vehicles $[1,19,20]$.

Considering design of a controller based on a transverse function one can find that its performance is strongly determined by properties of the chosen function. Hence, selection of a transverse function and its parametrization seems to be a critical issue. However, investigation of the transverse function properties can be difficult on highly dimensional domain. Then it is very hard to use analytic tools to describe global properties of the function. To overcome this issue we propose to use another methods based on discrete approach in order to approximate the function features which are not known analytically. We deal with probabilistic searching over whole domain and refer to Monte-Carlo analysis.

In particular we focus on control properties of the controller designed for motion control of unicycle-like vehicle pulling the set of trailers. From a theoretical point of view this paper is based on control solutions considered in [21] however it takes advantages of a more general description concerning $N \geq 1$ trailers. Here we deal with an optimal selection of transverse function parameters taking into account guaranteed tracking precision and the minimization of resultant gain in the control feedback.

We examine the robustness of the controller with respect to the additive bounded measurement noise. In order to get the quantitative measures extensive simulations are conducted with respect to a vehicle with one and two trailers. Based on the obtained results the conclusions considering possibility of the controller implementation in practice are formulated. According to best authors' knowledge such an analysis devoted to the control algorithms using transverse functions has not been considered in the literature.

The paper is organized as follows. In Section 2 model of control process is presented. Next section is an overview of the controller design. The control solutions, their analysis and methods of searching for properties of the open-control loop based on probabilistic algorithms are discussed in Section 4. The particular simulation results illustrating the controller performance applied for vehicle with one and two trailers are presented in Section 5. Section 6 concludes the paper.

\section{Model Description}

Let us consider kinematics of a unicycle-like tractor with $N$ trailers illustrated in Fig. 1. The 


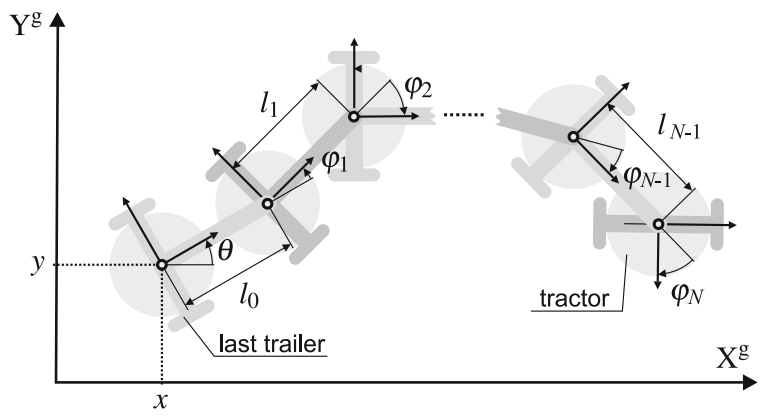

Fig. 1 Geometry of the considered vehicle

mechanical coordinates of the vehicle are given by $q:=\left[\begin{array}{llllll}x & y & \theta & \varphi_{1} & \ldots & \ldots\end{array} \varphi_{N}\right]^{\top} \in \mathcal{Q}:=\mathbb{R}^{2} \times \mathbb{T}^{N+1}$ (the symbol $\mathbb{T}^{k}$ is used to describe the $k$ th dimensional torus while $\mathbb{T}=\mathbb{R} / 2 \pi \mathbb{Z}$ with $\mathbb{Z}$ being the set of integers), where $x, y$, and $\theta$ denote the position of the last trailer and its orientation determined with respect to the inertial frame, respectively, while $\varphi_{1}, \ldots, \varphi_{N}$ refer to the internal configuration describing the orientation of each segment with respect to the previous one. The distances between the origins of the adjacent local frames fixed to each segment (trailer or tractor) are denoted by $l_{i}, i=0, \ldots, N-1$. Later in the paper it is assumed that feasible values of angles $\varphi_{j}, j=1,2, \ldots, N$, are limited and $q$ belongs to the restricted configuration set $\mathcal{Q}^{*}:=\mathbb{R}^{2} \times \mathbb{S}^{1} \times\left(-\frac{\pi}{2}, \frac{\pi}{2}\right)^{N} \subset \mathcal{Q}$. This property facilities the collision avoidance between the vehicle segments and ensures constant nonholonomy degree of the vehicle kinematics [8]. Following the given assumption we define auxiliary input

$v:=\left[\begin{array}{l}v_{1} \\ v_{2}\end{array}\right]=T(\varphi) v=\left[\begin{array}{cc}\prod_{i=1}^{N} \cos \varphi_{i} & 0 \\ -\frac{\sin \varphi_{N}}{l_{N-1}} & 1\end{array}\right] v$

with $v:=\left[\begin{array}{ll}v_{1} & v_{2}\end{array}\right]^{\top} \in \mathbb{R}^{2}$ denoting the linear, $v_{1}$, and angular, $v_{2}$, velocity of the tractor. Then for any $q \in \mathcal{Q}^{*}$ the kinematics of the considered vehicle can be described by the control system

$\Sigma: \dot{q}=X_{1}(q) v_{1}+X_{2} v_{2}$ with

$$
\begin{aligned}
X_{1}(q) & :=\left[\begin{array}{c}
\cos \theta \\
\sin \theta \\
\frac{\tan \varphi_{1}}{l_{0}} \\
\frac{l_{0} \tan \varphi_{2}-l_{1} \sin \varphi_{1}}{l_{0} l_{1} \cos \varphi_{1}} \\
\frac{l_{1} \tan \varphi_{3}-l_{2} \sin \varphi_{2}}{l_{1} l_{2} \cos \varphi_{1} \cos \varphi_{2}} \\
\vdots \\
\frac{l_{N-2} \tan \varphi_{N}-l_{N-1} \sin \varphi_{N-1}}{l_{N-2} l_{N-1} \prod_{i=1}^{N} \cos \varphi_{i}} \\
0 \\
X_{2}:=\left[\begin{array}{c}
0 \\
0 \\
0 \\
0 \\
0 \\
\vdots \\
1
\end{array}\right]
\end{array}\right],
\end{aligned}
$$

being basic vector fields.

\section{Preliminary Design of the Controller}

\subsection{Main Control Problem}

Consider a reference smooth trajectory $q_{r}:=$

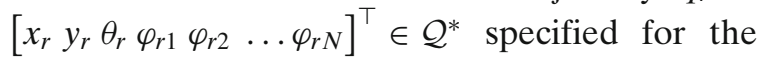
last trailer and internal configuration of system $\Sigma$. To quantify the control objective the following tracking error is defined $q_{e}=\left[\begin{array}{lll}x_{e} & y_{e} \theta_{e} & \varphi_{e 1} \varphi_{e 2} \ldots\end{array}\right.$ $\left.\ldots \varphi_{e N}\right]^{\top}:=q-q_{r}$. Then, the control problem can be stated as follows:

Problem 1 (Main control problem) Find bounded smooth kinematic control inputs $v_{1}$ and $v_{2}$ of the tractor such that for any smooth reference trajectory $q_{r}$ and $q_{e}(0) \in B_{0, \epsilon_{0}}$, where $B_{0, \epsilon_{0}}$ denotes the hyperball with center at the origin and radius $\epsilon_{0}>$ 0 , configuration error is bounded and asymptotically converges to the neighborhood with arbitrarily chosen radius $\epsilon>0$, i.e. $\sup _{t \geq 0}\left\|q_{e}(t)\right\|<\infty$, $\lim _{t \rightarrow \infty} q_{e}(t) \in B_{0, \epsilon}$, and configuration $q$ stays in 
the restricted configuration set, namely $q(0) \in$ $\mathcal{Q}^{*} \Rightarrow \forall t>0, q(t) \in \mathcal{Q}^{*}$.

In the definition of the control problem the practical stabilization paradigm is considered, namely it is assumed that the configuration error converges to some vicinity of zero with the selected radius. Theoretically, it gives possibility to approximate any smooth reference trajectory with arbitrarily chosen accuracy. Moreover, in some particular cases the possibility of asymptotic convergence is not excluded.

\subsection{Tracking Error}

To facilitate design of the control law it can be convenient to use another definition of tracking error than ordinary difference given by $q_{e}$. Basically, one can refer to Lie group theory to take advantage of some intrinsic symmetry in the configuration space.

Following this idea it is possible to consider kinematics on the last trailer as a system evolving on Lie group $G^{E} \sim S E(2)$ with the operation

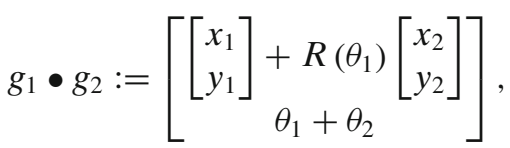

where $g_{i}:=\left[\begin{array}{ll}x_{i} & y_{i} \\ \theta_{i}\end{array}\right]^{\top} \in G^{E}$ for $i=1,2$ and $R \in$ $\mathrm{SO}(2)$, and neutral element $e:=\left[\begin{array}{lll}0 & 0 & 0\end{array}\right]^{\top}$. Consequently, denoting configuration of the trailer by

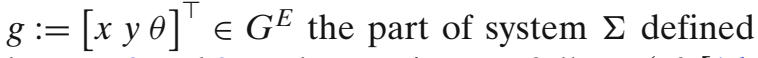
by Eqs. 2 and 3 can be rewritten as follows (cf. [16, 21])

$\Sigma_{g}: \dot{g}=X^{E}(g) C_{g}(\varphi) v$,

where

$C_{g}(\varphi):=\left[\begin{array}{cc}1 & 0 \\ 0 & 0 \\ \frac{\tan \varphi_{1}}{l_{0}} & 0\end{array}\right], X^{E}(g):=\left[\begin{array}{ccc}\cos \theta & -\sin \theta & 0 \\ \sin \theta & \cos \theta & 0 \\ 0 & 0 & 1\end{array}\right]$,

while columns of matrix $X^{E}(g)$ are vector fields constituting basis of Lie algebra $\mathfrak{g}$ of group $G^{E}$.
Taking advantage of subsystem $\Sigma_{g}$ invariance, the tracking error concerning the last trailer can be defined by

$\tilde{g}=\left[\begin{array}{lll}\tilde{x} & \tilde{y} & \tilde{\theta}\end{array}\right]^{\top}:=g_{r}^{-1} \bullet g$.

From now it is assumed that reference motion is governed by

$\dot{g}_{r}=X^{E}\left(g_{r}\right) u_{r}$,

where $u_{r}=\left[\begin{array}{lll}v_{r x} & v_{r y} & v_{r \theta}\end{array}\right]^{\top}$ is the reference signal with $v_{r x}, v_{r y}$ being the longitudinal and lateral velocities and $v_{r \theta}$ describing the angular velocity of the target expressed with respect to its local frame. Taking the time derivative of $\tilde{g}$ and utilizing the left-invariance property of system $\Sigma_{g}$ one can obtain (cf. [16, 21])

$\dot{\tilde{g}}=X^{E}(\tilde{g})\left(C_{\varphi}(\psi) v-A d^{X^{E}}\left(\tilde{g}^{-1}\right) u_{r}\right)$,

where $A d^{X^{E}}$ ( $g$ ) is the adjoint operator evaluated at point $g$ and expressed in basis $X^{E}$ of the Lie algebra.

Next, we come back to the latter part of system $\Sigma$ (cf. Eqs. 2 and 3) concerning the internal kinematics of the vehicle. To make notation to be consistent the kinematics can be rewritten as

$\Sigma_{\varphi}: \dot{\varphi}=C_{\varphi}(\varphi) \nu$,

where $\varphi:=\left[\begin{array}{llll}\varphi_{1} & \varphi_{2} & \ldots & \varphi_{N}\end{array}\right]^{\top}$ and

$C_{\varphi}(\varphi):=\left[\begin{array}{ccccc}X_{1,4} & X_{1,5} & \ldots & X_{1, N-1} & 0 \\ 0 & 0 & \ldots & 0 & 1\end{array}\right]^{\top}$

with $X_{1, i}$ denoting the $i$ th coordinate of vector field $X_{1}$. It should be emphasized that no particular symmetry can be found for subsystem $\Sigma_{\varphi}$. Hence, definition of tracking error with respect to internal angles based on Lie group cannot be applied. This issue implies some difficulty in control development which can be partially overcome by using coordinate and input transformation to another invariant control system.

\subsection{Selected Properties of Chained System}

The control solution considered in this paper is designed on the base of two input $M$ dimensional, 
or $(M-2)$ th order, chained system $\Sigma_{\xi}$ : $\dot{\xi}=$ $X_{1}^{c}(\xi) w_{1}+X_{2}^{c} w_{2}$, where $\xi=\left[\begin{array}{llll}\xi_{1} & \xi_{2} & \ldots & \xi_{M}\end{array}\right]^{\top}$ is the coordinate vector, $w_{1}$ and $w_{2}$ denote the control inputs, while $X_{1}^{c}$ and $X_{2}^{c}$ are vector fields given by $X_{1}^{c}(\xi):=\left[\begin{array}{llllll}1 & 0 & \xi_{2} & \xi_{3} & \ldots & \xi_{M}\end{array}\right]^{\top} \in \mathbb{R}^{M}$ and $X_{2}^{c}:=$ $\left[\begin{array}{lllll}0 & 1 & 0 & \ldots\end{array}\right]^{\top} \in \mathbb{R}^{M}$.

Such system is defined on homogeneous Lie group $G^{c}$ with operation given by

$(\xi \star \zeta)_{i}:= \begin{cases}\xi_{i}+\zeta_{i}, & \text { if } i=1,2 \\ \xi_{i}+\zeta_{i}+\sum_{j=2}^{i-1} \frac{\zeta_{1}^{i-j}}{(i-j) !} \xi_{j}, & \text { otherwise }\end{cases}$

where $\xi, \zeta \in G^{c}$ with $i$ denoting $i$ th coordinate of appropriate vector. Using matrix notation the basis of Lie algebra of the group can be defined by $X^{c}(\xi):=\left[\begin{array}{llll}X_{1}^{c} & X_{2}^{c} & \ldots & X_{M}^{c}\end{array}\right] \in$ $\mathbb{R}^{M \times M}$ with $X_{i+1}^{c}=\left[X_{i}^{c}, X_{1}^{c}\right], i=2,3, \ldots, M-1$ being Lie brackets. These vector fields are homogeneous and satisfy $X_{i}^{c}\left(\Delta_{\varepsilon}^{r} \xi\right)=\varepsilon^{d} \Delta_{\varepsilon}^{r} X_{i}^{c}(\xi)$, $i=1,2, \ldots, M$, where $\Delta_{\varepsilon}^{r}$ is dilation operator with $r=[1,1,2,3, \ldots, M-1]$ being weights vector, $d$ denoting homogeneity order and $\varepsilon>0$. Following this notation the dilation $\Delta_{\varepsilon}^{r}$ can be defined as follows: $\Delta_{\varepsilon}^{r} \xi:=D(\varepsilon) \xi$, where $D(\varepsilon):=$ $\operatorname{diag}\left\{\varepsilon, \varepsilon, \varepsilon^{2}, \varepsilon^{3}, \ldots, \varepsilon^{M-1}\right\} \in \mathbb{R}^{M \times M}$ is the diagonal matrix.

Along with dilation operator one can use homogeneous norm $\rho: \xi \in G^{c} \rightarrow \mathbb{R}_{+} \cup 0$ defined by: $\rho(\xi):=\left(\left|\xi_{1}\right|^{\kappa}+\left|\xi_{2}\right|^{\kappa}+\sum_{i=3}^{M}\left|\xi_{i}\right|^{\frac{\kappa}{i-1}}\right)^{\frac{1}{\kappa}}$, where $\kappa$ is some positive integer evenly divisible by each weight $r_{i}$ associated with the given dilation $\Delta_{\varepsilon}^{r}$. Most important property satisfied by the homogeneous norm is given by

$\rho(D(\varepsilon) \xi)=\varepsilon \rho(\xi)$

that relates the norm with the dilation.

\subsection{Open-Loop Dynamics of the Transformed Tracking Error}

Consider the coordinate map $\Phi: \mathbb{R}^{2} \times\left(-\frac{\pi}{2}, \frac{\pi}{2}\right) \times$ $\left(-\frac{\pi}{2}, \frac{\pi}{2}\right)^{N} \rightarrow \mathbb{R}^{N+3}$

$\xi:=\Phi(\tilde{g}, \varphi)$

$$
=\left[\Phi_{1}(\tilde{g}, \varphi) \Phi_{2}(\tilde{g}, \varphi) \ldots \Phi_{N+3}(\tilde{g}, \varphi)\right]^{\top},
$$

where $\Phi_{N+3}:=\tilde{y}, \Phi_{i}:=\frac{1}{\cos \theta} \frac{\partial \Phi_{i+1}}{\partial(\tilde{\tilde{g}}, \varphi)} X_{1}$ for $i=N+2$, $N+1, \ldots, 2$ and $\Phi_{1}:=\tilde{x}$. Next, let $w=$ $\left[w_{1} w_{2}\right]:=U(\tilde{\theta}, \varphi) v$ be a linear map $\mathbb{R}^{2} \rightarrow \mathbb{R}^{2}$, where

$$
\begin{aligned}
U & (\tilde{\theta}, \varphi) v \\
& =\left[\left(\frac{\partial \Phi_{2}(\tilde{g}, \varphi)}{\partial \tilde{g}} X^{E}(\tilde{g}) C_{g}(\varphi)+\frac{\partial \Phi_{2}(\tilde{g}, \varphi)}{\partial \varphi} C_{\varphi}(\varphi)\right) v\right] .
\end{aligned}
$$

Applying these transformations with respect to tracking error kinematics given by Eq. 8 for $u_{r} \equiv$ 0 (namely considering static case) along with subsystem $\Sigma_{\varphi}$ gives the $M:=N+3$ dimensional chained system $\Sigma_{\xi}$ which can be considered as the equivalent control system.

Remark 1 It is important to note that maps Eqs. 13 and 14 are diffeomorphisms defined locally and some restriction on the angles domain is imposed, namely the transformation is well defined for $q \in \mathcal{Q}^{*}$ and $|\tilde{\theta}|<\frac{\pi}{2}$. Consequently the feasible domain of tracking error is constrained.

Remark 2 It can be verified that for $\tilde{\theta}=0$ and $\varphi=$ 0 inverse of matrix $U(\tilde{\theta}, \varphi)$ satisfies

$U(0,0)^{-1}=\left[\begin{array}{cc}1 & 0 \\ 0 & \prod_{i=0}^{N} l_{i}\end{array}\right]$.

Next referring to Eq. 1 it follows that

$\tilde{\theta}=0, \varphi=0 \Rightarrow v_{1}=w_{1}, v_{2}=\prod_{i=0}^{N-1} l_{i} w_{2}$.

Hence, it can be concluded that transmission between control inputs $v_{2}$ and $w_{2}$ is strictly related to vehicle geometry. Namely the control effort increases along with increasing the distances between vehicle segments.

In the general case for $u_{r} \neq 0$ the transformed system becomes

$\dot{\xi}=X_{1}^{c}(\xi) w_{1}+X_{2}^{c} w_{2}+p\left(\tilde{g}, \varphi, u_{r}\right)$

where $p\left(\tilde{g}, \varphi, u_{r}\right)=-\frac{\partial \Phi(\tilde{g}, \varphi)}{\partial \tilde{g}} X^{E}(\tilde{g}) A d^{X^{E}}\left(\tilde{g}^{-1}\right) u_{r}$ denotes the drift. It is evident from Eq. 13 that $\xi$ 
does not reflect internal configuration error $\varphi_{e}$. To overcome this issue we take advantage of the fact that system $\Sigma_{\xi}$ evolves on Lie group $G^{c}$ and introduce transformed configuration tracking error

$\tilde{\xi}=\xi_{r}^{-1} \star \xi$,

where $\xi_{r}:=\Phi\left(0, \varphi_{r}\right)$ is transformed internal reference configuration $\varphi_{r}$. Then the open-loop dynamics governing evolution of $\tilde{\xi}$ becomes [21]

$\dot{\tilde{\xi}}=X^{c}(\tilde{\xi})\left(C_{\xi} w+\tilde{p}\right)$,

where

$C_{\xi}:=\left[\begin{array}{lllll}1 & 0 & 0 & \ldots & 0 \\ 0 & 1 & 0 & \ldots & 0\end{array}\right]^{\top} \in \mathbb{R}^{(N+3) \times 2}$,

$X^{c}$ denotes the basis of the Lie algebra associated with Lie group $G^{c}$ and $\tilde{p}=\left(X^{c}(\tilde{\xi})\right)^{-1}\left(d l_{\xi_{r}^{-1}}(\xi) p+\right.$ $\left.d r_{\xi}\left(\xi_{r}^{-1}\right) \frac{d}{d t} \xi_{r}^{-1}\right)$ is the drift (cf. [21]).

\section{Control Algorithm}

\subsection{Transverse Function}

The controller we focus on is based on the transverse function approach. In this particular case such a function is considered for $M$ dimensional two input chained system $\Sigma_{\xi}$ and satisfies the following definition.

Definition 1 Let $f: \mathbb{T}^{M-2} \rightarrow G^{c}$ be a smooth function which satisfies

$$
\begin{aligned}
\forall \alpha & \in \mathbb{T}^{M-2}, \operatorname{rank}\left[X_{1}^{c}(f(\alpha)) X_{2}^{c}(f(\alpha))-\frac{\partial f(\alpha)}{\partial \alpha}\right] \\
& =\operatorname{dim} G^{c}=M
\end{aligned}
$$

and $\forall \alpha \in \mathbb{T}^{k} f(\alpha, \varepsilon) \in B_{e, \epsilon}$. Then function $f$ is transverse with respect to vector fields of control system $\Sigma_{\xi}$.

Derivation of a transverse function for the given vector fields is not unique. For example one can consider harmonic basis of virtual inputs and calculate flow of differential equation defined by the properly selected vector fields from Lie algebra [11]. In particular this algorithm can be efficiently used for a control system defined on a Lie group. In the considered case the transverse function can be calculated using the following formula [13]

$$
f(\alpha):={ }^{M-2} f\left(\alpha_{M-2}\right) \star^{M-3} f\left(\alpha_{M-3}\right) \star \ldots \star^{1} f\left(\alpha_{1}\right)
$$

where

${ }^{i} f\left(\alpha_{i}\right)=\exp \left(X_{1}^{c} \beta_{i, 1} \sin \alpha_{i}+X_{i+1}^{c} \beta_{i, 2} \cos \alpha_{i}\right)$,

$i=1,2, \ldots, M-2$, are the basic components of the transverse function, $X_{i+1}^{c}$ is vector field defined in Section 3.3 and $\exp (\cdot)$ denotes exponential map (cf. $[13,21])$. It is assumed that functions ${ }^{i} f$ are parametrized by the set of $2 M-4$ coefficients $\beta_{i, j} \in \mathbb{R}$, where $j=1,2$-for the details the reader can refer to [21]. It should be emphasized that selection of these parameters is not arbitrary since transversality condition given by Eq. 20 has to be satisfied. This problem will be considered in Section 4.4 more thoroughly.

It can be proved that for any $\alpha \in \mathbb{T}^{M-2}$ function given by Eq. 21 cannot be shrinked to neutral element without violating transversality condition. To overcome this problem the following generalized transverse function can be considered [17]

$\bar{f}\left(\alpha, \alpha_{r}\right):=f\left(\alpha_{r}\right)^{-1} \star f(\alpha)$,

where $f(\alpha)$ is the transverse function (Eq. 21) and $\alpha_{r} \in \mathbb{T}^{M-2}$ is an auxiliary parameter. The generalized transverse function satisfies: $\lim _{\alpha \rightarrow \alpha_{r}} \bar{f}\left(\alpha, \alpha_{r}\right)=e$, namely there exists some $\alpha \in \mathbb{T}^{M-2}$ such that value of the function $\bar{f}$ coincides with the neutral element.

Another important property of a transverse function defined for any homogeneous system is possibility of its scaling using dilation operator (defined in Section 3.3) such that

$\bar{f}^{\varepsilon}:=D(\varepsilon) \bar{f}$,

is the scaled transverse function for any $\varepsilon>0$. The proof that $\bar{f}^{\varepsilon}$ satisfies the transversality condition is given in the Appendix (cf. also definition of transverse function presented in $[13,16])$. 
Next, we focus on derivative of transverse function $\bar{f}^{\varepsilon}$. Taking advantage of Lie algebra basis $X^{c}$ the time derivative of $\bar{f}^{\varepsilon}$ can be expressed as follows: $\dot{\overline{f^{\varepsilon}}}=X^{c}\left(\bar{f}^{\varepsilon}\right)\left(A_{\alpha} \dot{\alpha}+A_{\alpha_{r}} \dot{\alpha}_{r}+A_{\varepsilon} \dot{\varepsilon}\right)$, where $A_{\alpha}:=X^{c}\left(\bar{f}^{\varepsilon}\right)^{-1} \frac{\partial \bar{f}^{\varepsilon}}{\partial \alpha} \in \mathbb{R}^{M \times(M-2)}, \quad A_{\varepsilon}:=$ $X^{c}\left(\bar{f}^{\varepsilon}\right)^{-1} \frac{\partial \bar{f}^{\xi}}{\partial \varepsilon} \in \mathbb{R}^{M}$ and $A_{\alpha_{r}}:=X^{c}\left(\bar{f}^{\varepsilon}\right)^{-1} \frac{\partial \bar{f}^{\varepsilon}}{\partial \alpha_{r}} \in$ $\mathbb{R}^{M \times(M-2)}$. This notation can be used conveniently for checking the transversality condition. Then referring to Eq. 20 one has

$$
\left[X_{1}^{c}\left(\bar{f}^{\varepsilon}\right) X_{2}^{c}\left(\bar{f}^{\varepsilon}\right)-X^{c}\left(\bar{f}^{\varepsilon}\right) A_{\alpha}\right]=X^{c}\left(\bar{f}^{\varepsilon}\right) \bar{C}_{\xi},
$$

with

$$
\bar{C}_{\xi}:=\left[\begin{array}{ll}
C_{\xi} & -A_{1} \\
& -A_{2}
\end{array}\right] \in \mathbb{R}^{M \times M},
$$

where $A_{1} \in \mathbb{R}^{2 \times(M-2)}$ and $A_{2} \in \mathbb{R}^{(M-2) \times(M-2)}$ satisfies $\left[\begin{array}{ll}A_{1}^{\top} & A_{2}^{\top}\end{array}\right]=A_{\alpha}^{\top}$. It can be proved that the transversality condition holds if $\bar{C}_{\xi}$ is full rank matrix. Taking into account the particular structure of $C_{\xi}$ this is guaranteed when $\operatorname{det} A_{2} \neq 0$.

\subsection{Control Law}

The essence of the control algorithm we describe is to track the trajectory described by the transverse function. In the considered case the tracking error can be quantified by $z:=\tilde{\xi} \star \bar{f}^{\varepsilon}-1$. The open-loop auxiliary dynamics takes the following form

$\dot{z}=X^{c}(z) A d^{X^{c}}\left(\bar{f}^{\varepsilon}\right)\left(\bar{C}_{\xi} \bar{w}+\tilde{p}-A_{\varepsilon} \dot{\varepsilon}-A_{\alpha_{r}} \dot{\alpha}_{r}\right)$,

where $\bar{w}:=\left[w^{\top} \dot{\alpha}^{\top}\right]^{\top} \in \mathbb{R}^{M}$ is the extended control input with $\dot{\alpha}$ being the virtual input determining the evolution of the transverse functions.

Here we recall two control solutions presented in [21]. The first one is based on decoupling technique while the second one is designed referring to an optimal control paradigm.
Proposition 1 (Classic control feedback) The feedback given as follows

$\bar{w}=\bar{C}_{\xi}^{-1}\left(A d^{X^{c}}\left(\bar{f}^{\varepsilon}-1\right) X^{c}(z)^{-1} K z-p^{*}\right)$,

where $K \in \mathbb{R}^{M \times M}$ is a Hurwitz-stable matrix and $p^{*}:=\tilde{p}-A_{\varepsilon} \dot{\varepsilon}-A_{\alpha_{r}} \dot{\alpha}_{r}$, applied to system (27) ensures its exponential stabilization, namely

$\forall t \geq 0,\|z(t)\| \leq\|z(0)\| \exp (-c t)$,

where $c>0$ is dependent on the eigenvalues of matrix $K$, and practical stabilization such that

$\lim _{t \rightarrow \infty} \tilde{\xi}(t) \in B_{e, \epsilon}$,

where $\epsilon$ denotes the arbitrary small radius of the neighborhood of neutral element of the group.

Proposition 2 (Optimal control feedback) Let $J(\bar{v})=\frac{1}{2} \bar{v}^{\top} W_{1} \bar{v}$, where $\bar{v}:=\left[v^{\top} \dot{\alpha}^{\top}\right]^{\top} \in \mathbb{R}^{M}$ is the modified extended control input (cf. definition of $\bar{w}$ ) and $W_{1} \in \mathbb{R}^{M \times M}$ denotes a symmetric positive definite matrix. Next, define $H:=$ $X^{c}(z) A d^{X^{c}}\left(\bar{f}^{\varepsilon}\right), Q:=H \bar{C}_{\xi} \bar{T}$ with

$\bar{T}:=\left[\begin{array}{cc}U(\tilde{\theta}, \varphi) T(\varphi) & 0_{2 \times(M-2)} \\ 0_{(M-2) \times 2} & I_{(M-2) \times(M-2)}\end{array}\right]$

and assume that $v_{s}^{\mathrm{c}}:=-Q^{-1} W_{2} z, \quad v_{d}^{\mathrm{c}}:=$ $-\bar{T}^{-1} \bar{C}_{\xi}^{-1} p^{*}, \quad v_{s}^{o}:=-z^{\top} W_{2} z W_{1}^{-1} Q^{\top} z \quad$ and $v_{d}^{o}:=-z^{\top} H p^{*} W_{1}^{-1} Q^{\top} z$, where superscripts "s" and " $d$ " are used in order to describe the static and dynamic terms of the controller, and $W_{2} \in \mathbb{R}^{M \times M}$ denotes a positive definite gain matrix. The control law defined as follows

$\bar{v}=\frac{\lambda_{s}}{m+\lambda_{s}} v_{s}^{c}+\frac{1}{m+\lambda_{s}} v_{s}^{o}+\frac{\lambda_{d}}{m+\lambda_{d}} v_{d}^{c}+\frac{1}{m+\lambda_{d}} v_{d}^{o}$

with $\lambda_{s}$ and $\lambda_{d}>0$ being positive coefficients, and $m:=z^{\top} Q W_{1}^{-1} Q^{\top} z$, applied to system $\Sigma$ along with input transformation (Eq. 1) makes $z=e$ to be exponentially stable equilibrium point and guarantees optimal control effort at each time instant in the sense of minimization of the performance index $J(\bar{v})$ when $\lambda_{s}$ and $\lambda_{d} \rightarrow 0$. 
Remark 3 The first version of the controller gives possibility to calculate auxiliary input $w$ which can be transformed to the input of the vehicle using diffeomorphisms (1) and (14). For the second control law these transformations are directly included in the formula (31) in order to optimize the control effort.

\subsection{Static State Accuracy}

In the steady state the auxiliary tracking error $z$ converges to neutral element of group $G^{c}$. This implies that $\lim _{t \rightarrow \infty}\left(\tilde{\xi}(t)-\bar{f}^{\varepsilon}(t)=e\right)$. As a result the accuracy is dependent on radius of the neighborhood at which the transverse function is contained. According to Eq. 24 the error can be made arbitrarily small by scaling the transverse function using parameter $\varepsilon$. In the considered case to quantify the auxiliary tracking error one can refer to homogeneous norm-then the following relationship holds

$\lim _{t \rightarrow \infty} \rho(\tilde{\xi}(t))=\varepsilon \rho(\bar{f}(t))$.

It should be noticed that the homogeneous norm is based on intrinsic property of the Lie group on which the control system is defined and gives possibility to facilitate the calculation on the group and its Lie algebra. However, interpretation of this norm is less intuitive in comparison to classic Euclidean norm.

In particular it is an issue in the original configuration space when each coordinate has pure geometric meaning (for example it can describe the position or orientation error). To be more precise we consider how the transformed tracking error $\tilde{\xi}$ is translated into the original error in $\mathcal{Q}^{*}$. Calculating $\xi$ from Eq. 18 and using coordinate map in the steady state one has: $\xi=$ $\Phi\left(e, \varphi_{r}\right) \star \bar{f}^{\varepsilon}$. Following this result tracking error $\tilde{g}$ and internal configuration $\varphi$ of the vehicle satisfy

$\lim _{t \rightarrow \infty}\left(\left[\begin{array}{l}\tilde{g} \\ \varphi\end{array}\right]-\Phi^{-1}\left(\Phi\left(e, \varphi_{r}\right) \star \bar{f}^{\varepsilon}\right)\right)(t)=0$.

Finally, it can be shown that $\lim _{t \rightarrow \infty} q_{e}(t) \in B_{0, \epsilon}$, with

$\epsilon=\delta\left(\varphi_{r}\right)\left\|\bar{f}^{\varepsilon}\right\|$ being some positive constant, while $\delta\left(\varphi_{r}\right)$ is a nonlinear positive function that is dependent on the selected reference angles $\varphi_{r}$.

Remark 4 Comparing the tracking error in the original configuration space and the transformed tracking error one can find that the former is dependent not only on the norm of the transverse function but it is also affected by selection of the reference internal configuration of the vehicle. Namely, for $\varphi_{r i} \neq 0(i=1, \ldots, N)$ the error bound increases for the same transverse function $\bar{f}^{\varepsilon}$.

Additionally, we can consider asymptotic convergence of the tracking error. According to results presented in [16] this possibility can be conditionally achieved using the generalized transverse function defined by Eq. 23 for some class of reference feasible trajectory $q_{r}$ which persistently excites the closed control loop. Then, for the particular value of parameter $\alpha_{r}$ the following relationship can hold: $\lim _{t \rightarrow \infty} \alpha(t)=\alpha_{r}$ and $\lim _{t \rightarrow \infty} \bar{f}^{\varepsilon}(t)=e$.

Optionally, taking advantage of the generalized transverse function one can adjust the parameter $\alpha_{r}$ in order to limit norm of $\bar{f}^{\varepsilon}$ for the given value of $\varepsilon$.

\subsection{Selection of Transverse Function Parameters}

The selection of transverse function and its parametrization for the given control system is not unique. However, it can be realized that its properties have a significant impact not only on preservation of transversality condition but also on the controller performance. Unfortunately, problem of searching of global properties of transverse function is getting difficult for higher dimensional domain of the function.

Here we consider the transverse function $\bar{f}$ on $M$-2-dimensional torus. In order to examine its properties on the whole domain one can take into account its properties determined locally at each point $\alpha$ of the domain $\mathbb{T}^{M-2}$. However, such analytical examination in the continuous domain may be very tedious or even no possible-then one can refer to approximate method taking advantage of the domain discretization. Following this approach we assume that the domain $\mathbb{T}^{M-2}$ 
is discretized using probabilistic (Monte-Carlo) method and use the following algorithm

$\overline{\text { Algorithm } 1 \text { Probabilistic searching on } \mathbb{T}^{M-2}}$ domain

Step 1 Let $\mathcal{P}$ denote an empty list.

Step 2 Generate uniform random $n$ samples over $\mathbb{T}^{M-2}$ assuming that $i$ th sample is given by ${ }^{i} \alpha=\left[\alpha_{1} \alpha_{2} \ldots \alpha_{k}\right]$ where $\alpha_{j} \in U(0,2 \pi)$, $j=1,2, \ldots, \ldots, M-2$, while $U(\underline{\alpha}, \bar{\alpha})$ denotes uniform distribution on the inter$\operatorname{val}(\underline{\alpha}, \bar{\alpha}) \subset \mathbb{R}$.

Step 3 For each ${ }^{i} \alpha$ calculate the chosen properties $p$ of function $\bar{f}$ evaluated at point $\alpha={ }^{i} \alpha$ and save them on a list $\mathcal{P}$.

Step 4 Search for the list $\mathcal{P}$ to estimate the chosen properties of function $\bar{f}$ on the whole domain.

Remark 5 One should be aware that the quality of estimation increases when $n$ is made large enough. To be more specific for $n \rightarrow \infty$ probability of proper estimation of real but unknown function properties determined on the given domain approaches 1.

Selection of Function Parameters Based on formula given by Eq. 22 it follows that one should choose suitable set of parameters $\beta_{i, j}(i=$ $1,2, \ldots, M-2$ and $j=1,2)$ in order to satisfy the transversality condition. From now we say that such parameters are feasible. It should be noted that the selection of feasible parameters is getting more difficult when the dimension of the control system increases. Basically, for $M \geq 4$ it is hard to find explicit closed mathematical formula which define necessary conditions which should be satisfied by the parameters-cf. [15].

Instead of searching for the feasible parameters set in an analytical way a probabilistic approach can be used. To make the notation more clear we assume that $\beta:=$ $\left[\beta_{1,1} \beta_{1,2} \ldots \beta_{1, M-2} \beta_{2,1} \beta_{2,2} \ldots \beta_{2, M-2}\right] \in \mathbb{R}^{2(M-2)}$ denotes set of candidate parameters. To verify if the set of parameters is feasible one can check determinant of matrix $A_{2}$ on the domain $\mathbb{T}^{k}$.

Having sets of preliminary feasible parameters one can consider which one should be selected. To propose an objective criterion for a comparison we note that parameters $\beta$ determine an extreme norm of the transverse function and affect its derivative. Therefore, we assume that for comparison purposes the preliminary parameters should be scaled to ensure that some property of the transverse function remains unchanged. Here we can use the following scaling procedure based on dilation

$\beta_{i, 1}=\epsilon \beta_{i, 1}^{\prime}, \quad \beta_{i, 2}=\epsilon^{i} \beta_{i, 2}^{\prime}, i=1,2, \ldots, M-2$,

where $\epsilon>0$. It can be proved (cf. Appendix and proposition of transverse function in [13]) that for feasible set $\beta^{\prime}$ a new set $\beta$ is also feasible.

For example, one can assume that maximum Euclidean norm of the transverse function $\bar{f}$ calculated on the given domain is preserved for any $\beta$. However, such a constraint is quite difficult to satisfy taking into account that Euclidean norm of function $\bar{f}$ is not linearly dependent on $\epsilon$ using scaling given by Eq. 35 and for different values of $\epsilon$ maximum Euclidean norm of transverse function is met at different value of $\alpha$. Hence, we replaced Euclidean norm by homogeneous norm and assume that $\rho_{\max }=\max \left\{\rho\left(\left.\bar{f}\right|_{\beta^{\prime}}\right)\right\}$ denotes maximum homogeneous norm of the transverse function for the given set $\beta^{\prime}$. Then one can find a coefficient $\epsilon:=\frac{1}{\rho_{\max }}$ and calculate normalized parameters referring to Eq. 35 .

$\overline{\text { Algorithm } 2 \text { Probabilistic selection of feasible pa- }}$ rameters of TF

Step 1 Let $\mathcal{B}$ denote an empty list.

Step 2 Generate uniform random $m$ samples over $(0,1)^{2(M-2)} \subset \mathbb{R}^{2(M-2)}$ assuming that lth sample ${ }^{l} \beta^{\prime}$ is parametrized by ${ }^{l} \beta_{i, j}^{\prime} \in$ $U(0,1)$.

Step 3 For each ${ }^{l} \beta^{\prime}$ use Algorithm 1:

(A) if $\operatorname{det} A_{2} \leq 0$ for any ${ }^{i} \alpha$ stop searching on the TF domain

(B) if condition $\operatorname{det} A_{2}>0$ is satisfied on the whole domain find maximum norm $\rho_{\max }$ and calculate the normalized parameters ${ }^{l} \beta$ using relationship (35) and put ${ }^{l} \beta$ on list $\mathcal{B}$ 
Optimal Selection of Function Parameters During the optimal selection of transverse function parameters one can take into account the controller performance in the transient state. Such an issue should not be ignored especially when implementation of the controller is taken into account.

We focus on this problem more thoroughly by referring to fundamental form of the controller given by Proposition 1 and assuming that the drift term is zero. Then the control feedback becomes

$\bar{w}=P X^{c}(z)^{-1} K z$,

where

$P:=\bar{C}_{\xi}^{-1} A d^{X^{c}}\left(\bar{f}^{-1}\right) \in \mathbb{R}^{M \times M}$.

It can be easily concluded that the resultant gain of the open loop is scaled not only by gain matrix $K$ but it is also dependent on matrices $P$ and $X^{c}(z)$. From Eqs. 26 and 37 it follows that $P$ is parametrized by $\beta$.

In order to design the controller one can minimize the gain of the open loop assuming that the convergence rate and accuracy in the steady state are preserved. In the considered case we define optimization task as follows:

Problem 2 Find normalized parameters set $\beta \in \mathcal{B}$ for which maximum Frobenius norm of matrix $P$ denoted by $\|P\|_{F}$ becomes minimal.

It should be noticed that according to definition of Problem 2 the accuracy in the steady state is quantified using homogeneous norm since the normalized set of parameters is used. As a result the classical measure based on Euclidean metrics is not preserved. This assumption is a consequence of simplification which is made for searching of the normalized parameters (the main motivation is reduction of numerical complexity of the algorithm used for parameter normalization).

$\overline{\text { Algorithm } 3 \text { Selection of optimal parameter set of }}$ TF

Step 1 For each ${ }^{l} \beta \in \mathcal{B}$ use Algorithm 1 to calculate maximum norm ${ }^{l} \bar{\sigma}:=\left.\max \|P\|_{F}\right|_{\iota_{\beta}}$ on $\mathbb{T}^{M-2}$ domain.

Step 2 Find optimal parameter set $\beta_{\text {opt }}:={ }^{l} \beta$ for which ${ }^{l} \bar{\sigma}$ is minimal.
4.5 Robustness to Measurement Noise

Robustness of the control algorithm to the measurement noise is very important issue in practice. From a theoretical point of view the smooth control law considered here guarantee some robustness to unmodeled phenomena.

Based on analysis presented in Section 4.4 we examine how the function scaling affects the robustness of the algorithm. In order to do that we consider matrix $P$ defined by Eq. 37 assuming that the transverse function is dilated according to Eq. 24.

First, we take into account matrix $\bar{C}_{\xi}$ given by Eq. 26. Referring to Section 4.1 the term $A_{\alpha}$ can be calculated as follows

$A_{\alpha}=X^{c}(D(\varepsilon) \bar{f})^{-1} D(\varepsilon) \frac{\partial \bar{f}}{\partial \alpha}$.

Using the following relationship $X^{c}(D(\varepsilon) \bar{f})=$ $D(\varepsilon) X^{c}(\bar{f}) D(\varepsilon)^{-1}$ in Eq. 38 gives $A_{\alpha}=D(\varepsilon) \bar{A}_{\alpha}$, where $\bar{A}_{\alpha}:=X^{c}(\bar{f})^{-1} \frac{\partial \bar{f}}{\partial \alpha}$. Then matrix defined by Eq. 26 becomes

$\bar{C}_{\xi}=\left[C_{\xi} \mid-D(\varepsilon) \bar{A}_{\alpha}\right]=D(\varepsilon)\left[D(\varepsilon)^{-1} C_{\xi} \mid-\bar{A}_{\alpha}\right]$.

Calculating inverse of matrix $\bar{C}_{\xi}$ one obtains

$\bar{C}_{\xi}^{-1}=\varepsilon \bar{C}^{*} D(\varepsilon)^{-1}$,

with

$\bar{C}^{*}:=\left[\begin{array}{cc}I_{2 \times 2} & -A_{1} A_{2}^{-1} \\ 0_{(M-2) \times 2} & -\varepsilon^{-1} A_{2}^{-1}\end{array}\right]$,

while $A_{1} \in \mathbb{R}^{2 \times(M-2)}$ and $A_{2} \in \mathbb{R}^{(M-2) \times(M-2)}$ satisfies $\left[A_{1}^{\top} A_{2}^{\top}\right]=\bar{A}_{\alpha}^{\top}$.

Next, we investigate the second matrix in Eq. 37 taking into account the following relationships:

$$
\begin{aligned}
A d^{X^{c}}\left(\bar{f}^{\varepsilon}-1\right) & =A d^{X^{c}}\left(\bar{f}^{\varepsilon}\right)^{-1} \text { and } \\
A d^{X^{c}}(D(\varepsilon) \bar{f}) & =D(\varepsilon) A d^{X^{c}}(\bar{f}) D(\varepsilon)^{-1} .
\end{aligned}
$$

Then using Eqs. 40 and 37 leads to

$$
P=\varepsilon \bar{C}^{*} A d^{X^{c}}(\bar{f})^{-1} D(\varepsilon)^{-1} .
$$


The Frobenius norm of $P$ can be estimated as follows

$$
\|P\|_{F} \leq c\left\|\bar{C}^{*}\right\|_{F}\left\|\varepsilon D(\varepsilon)^{-1}\right\|_{F},
$$

where $c:=\left\|A d^{X^{c}}(\bar{f})^{-1}\right\|$ is the term independent on $\varepsilon$,

$$
\left\|\bar{C}^{*}\right\|_{F}=\varepsilon^{-1} \sqrt{\varepsilon^{2}\left\|\left[I_{2 \times 2}-A_{1} A_{2}^{-1}\right]\right\|_{F}^{2}+\left\|A_{2}^{-1}\right\|_{F}^{2}}
$$

and $\left\|\varepsilon D(\varepsilon)^{-1}\right\|_{F}=\sqrt{2+\sum_{i=1}^{M-2} \varepsilon^{-2 i}}$. Additionally the following limits can be considered:

$$
\begin{aligned}
\varepsilon & \rightarrow \infty \Longrightarrow\left\|\bar{C}^{*}\right\|_{F} \rightarrow\left\|\left[I_{2 \times 2}-A_{1} A_{2}^{-1}\right]\right\|_{F}, \\
& \left\|\varepsilon D(\varepsilon)^{-1}\right\|_{F} \rightarrow \sqrt{2}, \\
\varepsilon & \rightarrow 0^{+} \Longrightarrow\left\|\bar{C}^{*}\right\|_{F} \rightarrow \varepsilon^{-1}\left\|A_{2}^{-1}\right\|_{F}, \\
& \left\|\varepsilon D(\varepsilon)^{-1}\right\|_{F} \rightarrow \varepsilon^{-M+2}
\end{aligned}
$$

Following these results it can be concluded that $\|P\|_{F}$ is bounded for $\varepsilon \rightarrow \infty$ and it is unbounded when $\varepsilon$ approaches zero. As a result parameter $\varepsilon$ gives possibility to change resultant gain of the controller. This property is essential to guarantee robustness of the controller. Namely, in the case when some unmodeled phenomena (for example: measurement noise) is present one should increase value of $\varepsilon$.

On the other hand, increasing $\varepsilon$ leads to the relaxation of tracking accuracy which may not be acceptable in practice. To overcome this drawback one can refer to the generalized transverse function and use free parameter $\alpha_{r}$ in order to follow (at least approximately) current auxiliary state $\alpha$. It can be quite easily realized when initial tracking error $\tilde{\xi}(0)=e$-then one can set $\alpha(0)=\alpha_{r}$. Moreover, considering tracking of persistence exciting reference trajectories for the chained system it is recommended to set $\alpha_{r}=-\left[\begin{array}{llll}\frac{\pi}{2} & \frac{\pi}{2} & \ldots & \frac{\pi}{2}\end{array}\right]^{\top}$ - the details can be found in [16, 17].

\section{Simulation Results}

Here we consider two particular cases for $N=1$ and $N=2$ taking into account classic and generalized transverse functions and using algorithms described in Section 4.4. The calculations were performed in Matlab environment using Parallel Computing Toolbox in order to speed up searching algorithms.

\subsection{Selection of Transverse Functions Parameters}

First, we focus on selection of transverse function separately. In order to do this we considered $m=10^{4}$ candidates ${ }^{l} \beta$ for $N=1$. For each ${ }^{l} \beta$ the domain of the transverse function was sampled with $n=4 \cdot 10^{4}$ samples ${ }^{i} \alpha$ using Algorithms 1 and 2. For $N=2$ the amount of samples were chosen as $m=10^{4}$ and $n=10^{5}$, respectively. As a result, 3,360 and 392 feasible normalized parameters in $\mathcal{B}$ were found for $N=1$ and $N=2$. The normalization was done separately with respect to classic and generalized transverse functions defined by Eqs. 21 and 23. Hereafter, these functions are abbreviated as CTF and GTF, respectively.

Next, maximum Frobenius norm of matrix $P$ for each set of parameters in $\mathcal{B}$ was calculated for the considered cases. In Fig. 2 values of norm are presented while the data are sorted in ascending order for better clarity. Analyzing these graphs it can be concluded that the matrix norm is changed in a significant range-especially it is evident for $N=2$. It proves that different selection of feasible parameters $\beta$ effects the control feedback considerably.

The optimal sets of parameters were chosen taking into account minimization of $\max \|P\|_{F}$. The optimal parameters values are collected in Table 1. Next, the proper set of optimal parameter was selected for the given transverse function (including classic TF or generalized TF defined for $N=1$ and $N=2$ ).

It is important to note that Frobenious norm of matrix $P$ changes on domain $\mathbb{T}^{k}$. It can be noticed from Fig. 3 that extreme values of the norm can vary by several orders of magnitude. Hence, the open loop gain is strictly dependent on current value of variable $\alpha$. It can be noticed that the norm increases along with control system dimension and becomes higher when the generalized TFs are considered. 
Fig. 2 Maximum Frobenious norm of matrix $P$ calculated for the normalized parameters for $N=1$ and $N=2$

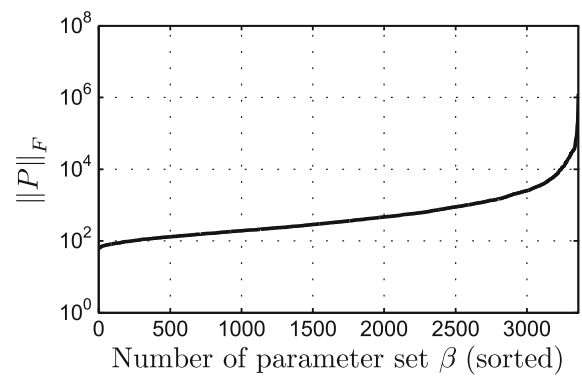

(a) $\mathrm{CTF}, N=1$

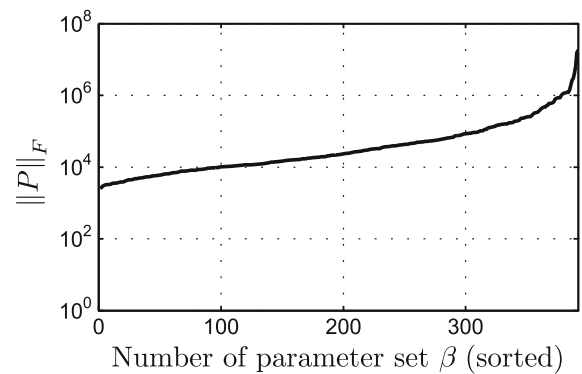

(c) $\mathrm{CTF}, N=2$

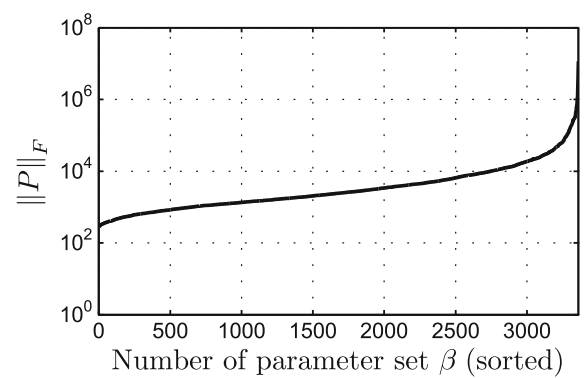

(b) GTF, $N=1$

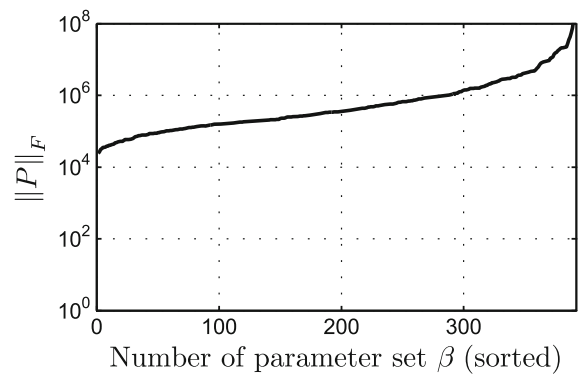

(d) GTF, $N=2$
5.2 Examination of Properties of Closed-Loop Control System

Next, we come to examination how the TFs determine performance of the closed-loop control system. The detailed analysis is presented assuming that the geometrical parameters of the vehicle are given by: $l_{0}=l_{1}=1 \mathrm{~m}$.

Static State Accuracy The static accuracy is verified considering tracking of constant reference trajectory (namely stabilization at the neighborhood of $q_{r}$ ). The upper bound of each coordinate of vector $q_{e}$ is calculated for $\varepsilon \in[0.001,10]$. The calculations are performed for $\varphi_{r}=0$ and $\varphi \neq 0$ taking into account classical and generalized TFs with parameters $\beta$ collected in Table 1 . The results are presented in Figs. 4, 5, 6 and 7. It should be mentioned that the configuration errors in the particular cases in the steady-state may be less than specified by the bounds. For example, since the generalized TF gives possibility to render the origin point $q=q_{r}$ can be made an equilibrium point.

From the given results it can be concluded that $\varepsilon$ mostly affects error $\tilde{y}$. This is due to the property of coordinate transformation $\Phi$ (notice that the last transformed state $\xi_{N-2}=\tilde{y}$ ) and the dilation operator $\Delta_{\varepsilon}^{r}$ that scales elements of vector $\tilde{\xi}$ according to weights given by $r$. For the same reason maximum bound of error $\tilde{x}$ is linearly dependent on $\varepsilon$. It is worth to comment that angular errors behave differently in comparison to position errors. Namely, the bounds of angular errors
Table 1 Optimal parameters of $\mathrm{TF}$

\begin{tabular}{llllll}
\hline & \multicolumn{3}{l}{ Trailers number } & & \\
\cline { 2 - 3 } & $N=1$ & & & $N=2$ & \\
\cline { 2 - 3 } \cline { 5 - 6 } Parameters & Classic TF & Generalized TF & & Classic TF & Generalized TF \\
\hline$\beta_{1,1}$ & 0.256157893 & 0.121138497 & & 0.100423025 & 0.052109198 \\
$\beta_{1,2}$ & 0.953738102 & 0.891623063 & & 0.925327028 & 0.939410985 \\
$\beta_{2,1}$ & 0.743845288 & 0.378864310 & & 0.294256612 & 0.150747282 \\
$\beta_{2,2}$ & 0.509203554 & 0.413066795 & & 0.170280638 & 0.092588245 \\
$\beta_{3,1}$ & - & - & 0.605369661 & 0.296884259 \\
$\beta_{3,2}$ & - & - & 0.166532578 & 0.044430930 \\
\hline
\end{tabular}


Fig. 3 Frobenius norm of matrix $P$ for the optimal parameters-for $N=1$ and $N=2$

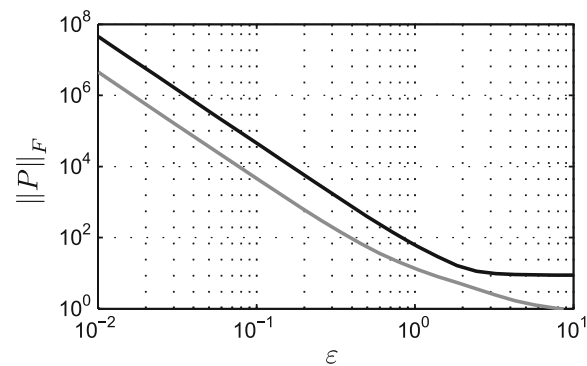

(a) $\mathrm{CTF}, N=1$

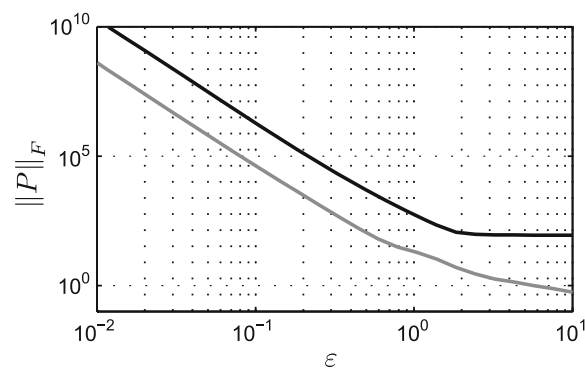

(c) $\mathrm{CTF}, N=2$

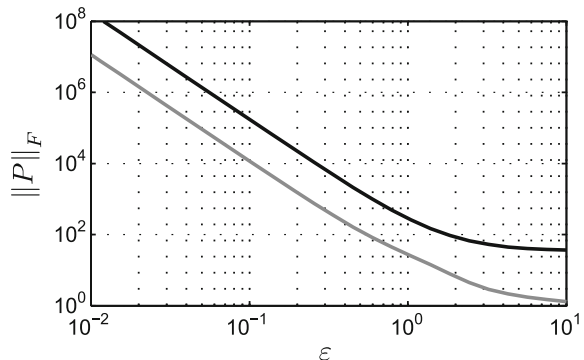

(b) GTF, $N=1$

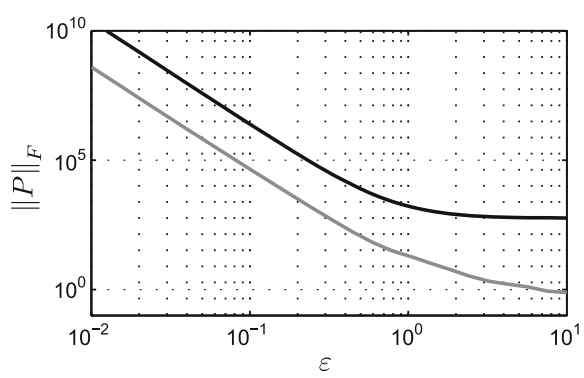

(d) GTF, $N=2$ are clearly limited. This property comes from a local nature of map $\Phi$. Hence, for higher value of $\varepsilon$ the bounds of angular errors almost correspond to bounds imposed by the map domain. Consequently, when $\varepsilon$ increases the guaranteed accuracy of the controller with respect to angular measures declines rapidly. Another drawback of the transformation can be seen for significant magnitude of $\varphi_{r i}$. In such a case the error bounds increase considerably, in particular for higher $\mathrm{N}$ -
Fig. 4 Error bounds in the steady state for $N=1$ and $\varphi_{r}=0$

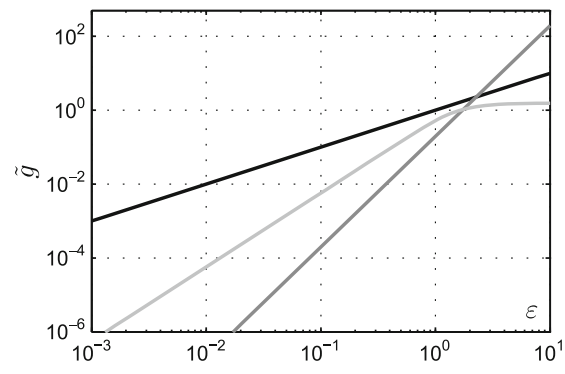

(a) CTF, errors: $\tilde{x} \square, \tilde{y} \square[\mathrm{m}], \tilde{\theta} \square[\mathrm{rad}]$

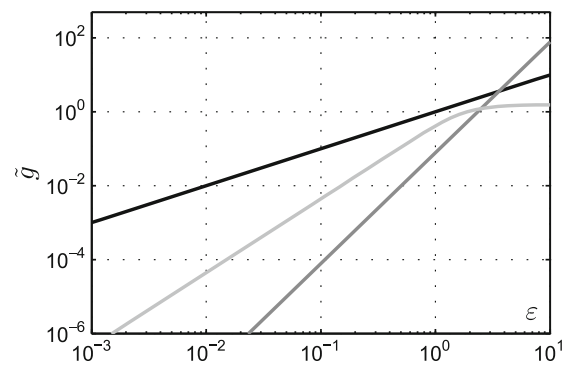

(c) GTF, errors: $\tilde{x} \square, \tilde{y} \square[\mathrm{m}], \tilde{\theta} \square[\mathrm{rad}]$

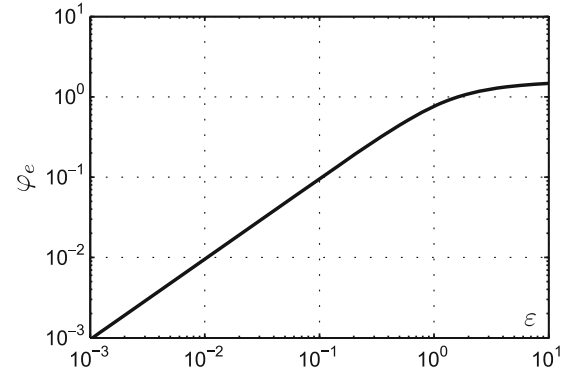

(b) CTF, errors: $\varphi_{e 1} \mathbf{\square}[\mathrm{rad}]$

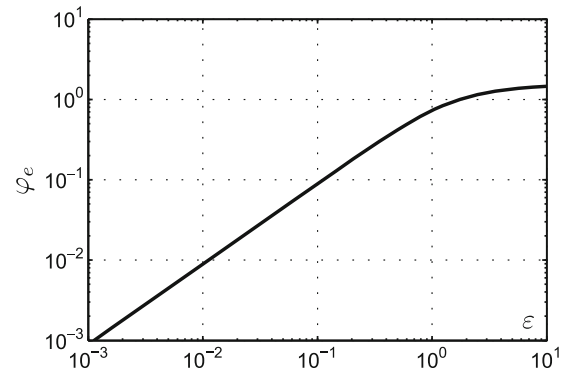

(d) GTF, errors: $\varphi_{e 1} \boldsymbol{\square}[\mathrm{rad}]$ 
Fig. 5 Error bounds in the steady state for $N=1, \varphi_{r}=\pi / 3 \mathrm{rad}$

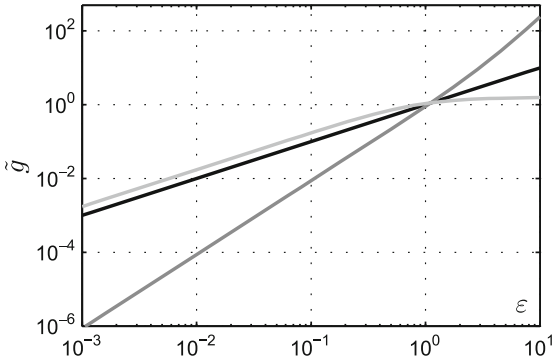

(a) CTF, errors: $\tilde{x} \square, \tilde{y} \square[\mathrm{m}], \tilde{\theta} \square[\mathrm{rad}]$

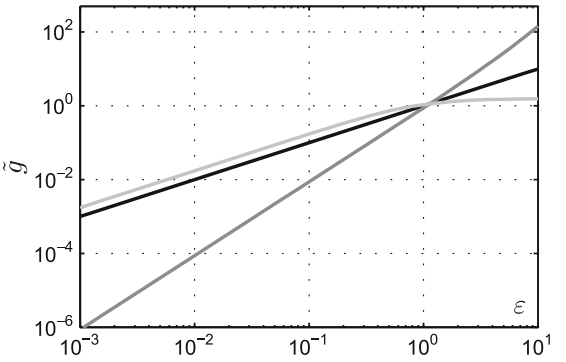

(c) GTF, errors: $\tilde{x} \square, \tilde{y} \square[\mathrm{m}], \tilde{\theta} \square[\mathrm{rad}]$

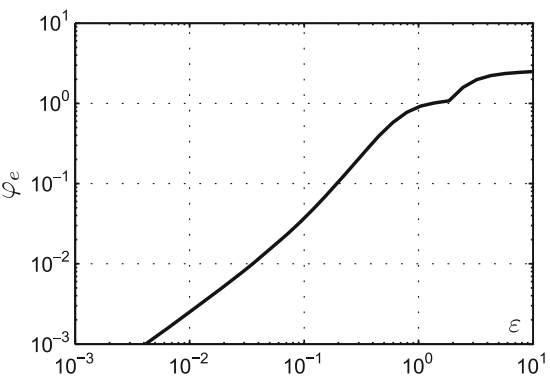

(b) CTF, errors: $\varphi_{e 1} \mathbf{\square}[\mathrm{rad}]$

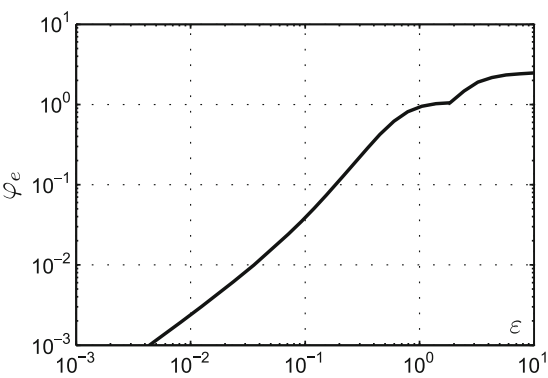

(d) GTF, errors: $\varphi_{e 1} \mathbf{\square}[\mathrm{rad}]$ cf. Figs. 5 and 7. Comparing the results obtained for classic and generalized TFs one can state that selection of function type does not affect error bound significantly (most visible difference can be found with respect to error $\tilde{y}$ ).
Robustness to the Noise-Static Case Next research problem is focused on robustness issue. In order to make the simulations it was assumed that the position and orientation measurements are affected by an additive uniformly distributed
Fig. 6 Error bounds in the steady state for $N=2$ and $\varphi_{r}=0$
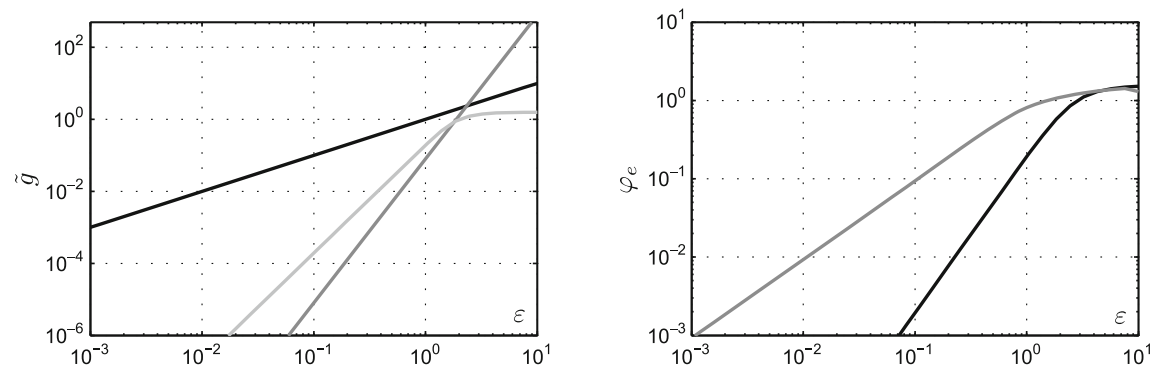

(a) CTF, errors: $\tilde{x} \square, \tilde{y} \square[\mathrm{m}], \tilde{\theta} \square[\mathrm{rad}]$

(b) CTF, errors: $\varphi_{e 1} \mathbf{\square}, \varphi_{e 2} \square[\mathrm{rad}]$

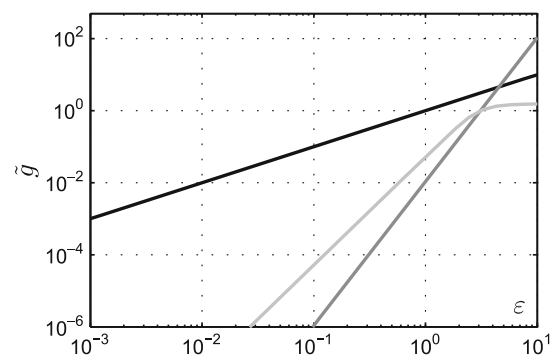

(c) GTF, errors: $\tilde{x} \square, \tilde{y} \square[\mathrm{m}], \tilde{\theta} \square$ [rad]

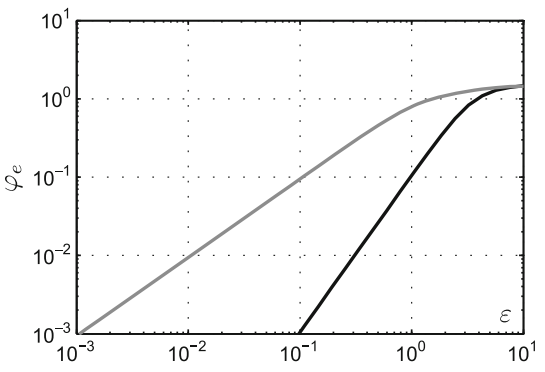

(d) GTF, errors: $\varphi_{e 1} \mathbf{a}, \varphi_{e 2} \square[\mathrm{rad}]$ 
Fig. 7 Error bounds in the steady state for $N=2$ and $\varphi_{r}=[-\pi / 3 \pi / 3]^{\top}$
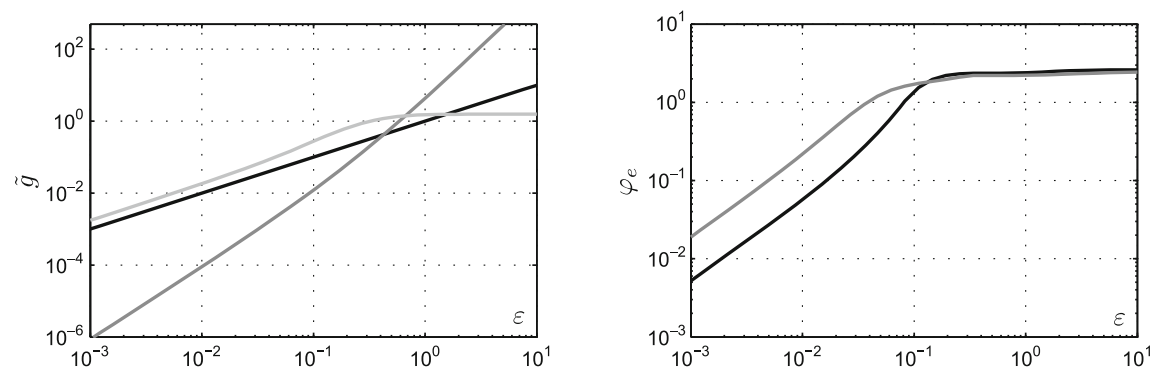

(a) CTF, errors: $\tilde{x} \square, \tilde{y} \square[\mathrm{m}], \tilde{\theta} \square[\mathrm{rad}]$

(b) CTF, errors: $\varphi_{e 1} \boldsymbol{\square}, \varphi_{e 2} \square[\mathrm{rad}]$

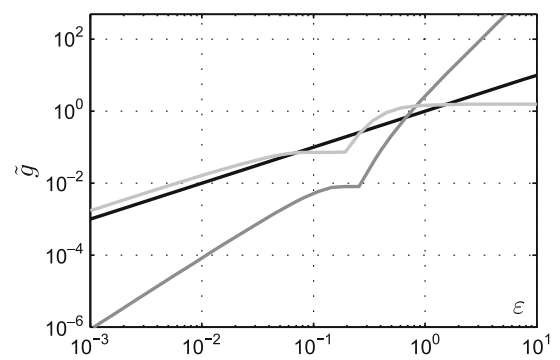

(c) GTF, errors: $\tilde{x} \square, \tilde{y} \square[\mathrm{m}], \tilde{\theta} \square[\mathrm{rad}]$

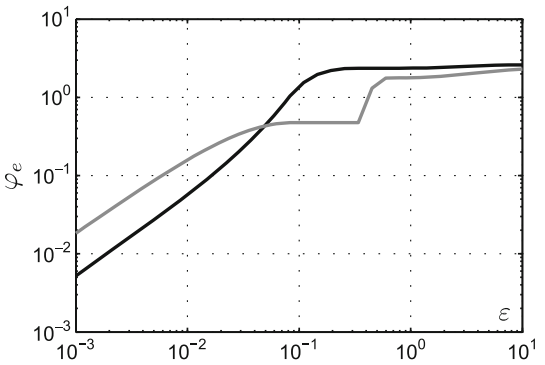

(d) GTF, errors: $\varphi_{e 1} \square, \varphi_{e 2} \square[\mathrm{rad}]$ uncorrelated noise. The parameters of the noise were selected as follows: $\delta_{x}, \delta_{y} \in U(-0.005,0.005)$ and $\delta_{\theta} \in U(-0.02,0.02)$ (such noise can be met in practice-cf. [4]). To verify sensitivity of the closed-loop control system to the noise simulations were performed in Simulink environment. The controller based on Proposition 1 was used with gain matrix $K=-I_{(N+3) \times(N+3)}$, where $I$ denotes identity matrix. It was assumed that $\varepsilon=$ const, $\alpha_{r}=\alpha$ (in the case of generalized TF) and $q_{e}(0)=0$ while $q_{r}=$ const. The simulations were repeated 100 times using randomly chosen $\alpha(0)$ with time horizon $t_{H}=20 \mathrm{~s}$ for each selected value $\varepsilon$. In order to neglect transient states for the analysis purposes the samples from last 10 second were chosen. Then for each data the fundamental measures were calculated such as mean error values, standard deviation of error values and control input signals. Next, for the chosen $\varepsilon$ maximum measures were found taking into account 100 results. Additionally, the minimum standard deviation concerning input $v_{2}$ was searched for and the corresponding initial condition $\alpha^{*}=\alpha(0)$ was recorded. The re-
Table 2 Results of simulations for $N=1$-classic TF, $\varphi_{r}=0$

\begin{tabular}{lllll}
\hline Measure & $\varepsilon=0.25$ & $\varepsilon=0.5$ & $\varepsilon=1$ & $\varepsilon=2$ \\
\hline $\max \operatorname{mean}\left(e_{x}\right)[\mathrm{m}]$ & 0.2178 & 0.3365 & 0.8693 & 1.9762 \\
$\max \operatorname{mean}\left(e_{y}\right)[\mathrm{m}]$ & 0.0031 & 0.0211 & 0.1727 & 1.3996 \\
$\max \operatorname{mean}\left(e_{\theta}\right)[\mathrm{rad}]$ & 0.0346 & 0.1375 & 0.5063 & 1.1476 \\
$\max \operatorname{mean}\left(e_{\varphi}\right)[\mathrm{rad}]$ & 0.2323 & 0.4343 & 0.7468 & 1.0813 \\
$\max \operatorname{std}\left(e_{x}\right)[\mathrm{m}]$ & 0.0198 & 0.0066 & 0.0022 & 0.0031 \\
$\max \operatorname{std}\left(e_{y}\right)[\mathrm{m}]$ & 0.0005 & 0.0006 & 0.0010 & 0.0063 \\
$\max \operatorname{std}\left(e_{\theta}\right)[\mathrm{rad}]$ & 0.0044 & 0.0031 & 0.0018 & 0.0014 \\
$\max \operatorname{std}\left(e_{\varphi}\right)[\mathrm{rad}]$ & 0.1092 & 0.0324 & 0.0088 & 0.0026 \\
$\max \operatorname{std}\left(v_{1}\right)[\mathrm{m} / \mathrm{s}]$ & 0.3154 & 0.1044 & 0.0477 & 0.1445 \\
$\max \operatorname{std}\left(v_{2}\right)[\mathrm{rad} / \mathrm{s}]$ & 1.6910 & 0.4585 & 0.1255 & 0.0398 \\
$\left.\operatorname{std}\left(v_{1}\right)\right|_{\alpha^{*}}[\mathrm{~m} / \mathrm{s}]$ & 0.2047 & 0.0949 & 0.0387 & 0.0248 \\
$\left.\operatorname{std}\left(v_{2}\right)\right|_{\alpha^{*}}[\mathrm{rad} / \mathrm{s}]$ & 0.0591 & 0.0351 & 0.0029 & 0.0037 \\
\hline
\end{tabular}


Table 3 Results of simulations for $N=1$-generalized TF, $\varphi_{r}=0$

\begin{tabular}{lllll}
\hline Measure & $\varepsilon=0.25$ & $\varepsilon=0.5$ & $\varepsilon=1$ & $\varepsilon=2$ \\
\hline $\max \operatorname{mean}\left(e_{x}\right)[\mathrm{m}]$ & 0.0339 & 0.0085 & 0.0024 & 0.0008 \\
$\max \operatorname{mean}\left(e_{y}\right)[\mathrm{m}]$ & 0.0001 & $4 \cdot 10^{-6}$ & $1.1 \cdot 10^{-7}$ & $5.5 \cdot 10^{-9}$ \\
$\max \operatorname{mean}\left(e_{\theta}\right)[\mathrm{rad}]$ & 0.0049 & 0.0006 & $8.8 \cdot 10^{-5}$ & $1.5 \cdot 10^{-9}$ \\
$\max \operatorname{mean}\left(e_{\varphi}\right)[\mathrm{rad}]$ & 0.3229 & 0.0859 & 0.0239 & 0.0066 \\
$\max \operatorname{std}\left(e_{x}\right)[\mathrm{m}]$ & 0.0262 & 0.0113 & 0.0031 & 0.0010 \\
$\max \operatorname{std}\left(e_{y}\right)[\mathrm{m}]$ & 0.0002 & $9 \cdot 10^{-6}$ & $2 \cdot 10^{-7}$ & $8.6 \cdot 10^{-9}$ \\
$\max \operatorname{std}\left(e_{\theta}\right)[\mathrm{rad}]$ & 0.0077 & $1 \cdot 10^{-3}$ & $6 \cdot 10^{-5}$ & $4.4 \cdot 10^{-6}$ \\
$\max \operatorname{std}\left(e_{\varphi}\right)[\mathrm{rad}]$ & 0.1598 & 0.1127 & 0.0321 & 0.0089 \\
$\max \max \operatorname{std}\left(v_{1}\right)[\mathrm{m} / \mathrm{s}]$ & 0.5389 & 0.1642 & 0.0462 & 0.0168 \\
$\max \max \operatorname{std}\left(v_{2}\right)[\mathrm{rad} / \mathrm{s}]$ & 4.8555 & 1.6595 & 0.4574 & 0.1265 \\
$\left.\operatorname{std}\left(v_{1}\right)\right|_{\alpha^{*}}[\mathrm{~m} / \mathrm{s}]$ & 0.2451 & 0.0529 & 0.0450 & 0.0162 \\
$\left.\operatorname{std}\left(v_{2}\right)\right|_{\alpha^{*}}[\mathrm{rad} / \mathrm{s}]$ & 0.4270 & 0.0615 & 0.0096 & 0.0034 \\
\hline
\end{tabular}

sults for the different scenarios are collected in Tables 2, 3, 4, 5, 6 and 7 .

Referring to the obtained results one can observe that it is difficult to guarantee small configuration error $q_{e}$ when classic TF is used. This is a result of high sensitivity of the closedcontrol loop to the unmodelled dynamics when $\varepsilon$ becomes small. In particular it is an issue with respect to error coordinate $\varphi_{e N}$ for which a significant variation can be noticed. The results confirm that the controller robustness to the noise increases along with $\varepsilon$. However, this parameter cannot be made too large because it may lead to significant configuration error which may be unacceptable from a practical point of view. It should be recalled that for the classic TF the minimum error bound is strictly dependent on $\varepsilon$. Hence, one can meet a relevant contradiction between the desired accuracy and the sensitivity of the controller.
Taking into account these aspects the better results can be achieved by using generalized TFs. According to simulation results it can be observed that the coordinate errors do not leave small neighborhood of zero for any $\varepsilon$. This property comes from the fact that initially it has been assumed that $\alpha(0)=\alpha_{r}$ and the noise with zero mean value was used. It should be emphasized that none of the simulations performed with generalized TF (1,200 tries) indicates that the there is the tendency to error increase. On the other hand theoretically there is no guarantee that $\alpha$ stays at $\alpha_{r}$-as a result $q_{e}=0$ is not the unique equilibrium for the regulation case.

Comparing standard deviation calculated for input signals it can be concluded that for the same $\varepsilon$ the robustness to the noise is higher when classic TF is used. However, this conclusion may not be true in the particular cases. For example, referring to Tables 5 and 6 one can find find that for $\varepsilon=$
Table 4 Results of simulations for $N=1$-generalized TF, $\varphi_{r}=\pi / 3$

\begin{tabular}{lllll}
\hline Measure & $\varepsilon=0.25$ & $\varepsilon=0.5$ & $\varepsilon=1$ & $\varepsilon=2$ \\
\hline $\max \operatorname{mean}\left(e_{x}\right)[\mathrm{m}]$ & 0.0344 & 0.0086 & 0.0025 & 0.0008 \\
$\max \operatorname{mean}\left(e_{y}\right)[\mathrm{m}]$ & 0.0007 & 0.0001 & $1 \cdot 10^{-5}$ & $1.9 \cdot 10^{-6}$ \\
$\max \operatorname{mean}\left(e_{\theta}\right)[\mathrm{rad}]$ & 0.0348 & 0.0087 & 0.0026 & 0.0009 \\
$\max \operatorname{mean}\left(e_{\varphi}\right)[\mathrm{rad}]$ & 0.1896 & 0.0458 & 0.0119 & 0.0034 \\
$\max \operatorname{std}\left(e_{x}\right)[\mathrm{m}]$ & 0.0263 & 0.0112 & 0.0032 & 0.0011 \\
$\max \operatorname{std}\left(e_{y}\right)[\mathrm{m}]$ & 0.0010 & 0.0001 & $1 \cdot 10^{-5}$ & $1.2 \cdot 10^{-6}$ \\
$\max \operatorname{std}\left(e_{\theta}\right)[\mathrm{rad}]$ & 0.0298 & 0.0114 & 0.0034 & 0.0012 \\
$\max \operatorname{std}\left(e_{\varphi}\right)[\mathrm{rad}]$ & 0.0946 & 0.0598 & 0.0158 & 0.0043 \\
$\max \operatorname{std}\left(v_{1}\right)[\mathrm{m} / \mathrm{s}]$ & 0.7260 & 0.2352 & 0.0681 & 0.0262 \\
$\max \operatorname{std}\left(v_{2}\right)[\mathrm{rad} / \mathrm{s}]$ & 2.6967 & 0.9078 & 0.2262 & 0.0643 \\
$\left.\operatorname{std}\left(v_{1}\right)\right|_{\alpha^{*}}[\mathrm{~m} / \mathrm{s}]$ & 0.2483 & 0.1466 & 0.0582 & 0.0212 \\
$\left.\operatorname{std}\left(v_{2}\right)\right|_{\alpha^{*}}[\mathrm{rad} / \mathrm{s}]$ & 0.3450 & 0.0310 & 0.0062 & 0.0034 \\
\hline
\end{tabular}


Table 5 Results of simulations for $N=2$-classic TF, $\varphi_{r}=0$

\begin{tabular}{lrllr}
\hline Measure & $\varepsilon=0.5$ & $\varepsilon=1$ & $\varepsilon=2$ & $\varepsilon=4$ \\
\hline $\max \operatorname{mean}\left(e_{x}\right)[\mathrm{m}]$ & 0.4243 & 0.8507 & 1.5505 & 3.5998 \\
$\max \operatorname{mean}\left(e_{y}\right)[\mathrm{m}]$ & 0.0044 & 0.0754 & 1.1632 & 19.0822 \\
$\max \operatorname{mean}\left(e_{\theta}\right)[\mathrm{rad}]$ & 0.0217 & 0.1758 & 0.9328 & 1.4699 \\
$\max \operatorname{mean}\left(e_{\varphi_{1}}\right)[\mathrm{rad}]$ & 0.0427 & 0.1890 & 0.6552 & 0.9105 \\
$\max \operatorname{mean}\left(e_{\varphi_{2}}\right)[\mathrm{rad}]$ & 0.3660 & 0.7737 & 1.0632 & 1.2953 \\
$\max \operatorname{std}\left(e_{x}\right)[\mathrm{m}]$ & 0.0738 & 0.0202 & 0.0053 & 0.1300 \\
$\max \operatorname{std}\left(e_{y}\right)[\mathrm{m}]$ & 0.0008 & 0.0020 & 0.0063 & 1.1844 \\
$\max \operatorname{std}\left(e_{\theta}\right)[\mathrm{rad}]$ & 0.0021 & 0.0025 & 0.0019 & 0.0031 \\
$\max \operatorname{std}\left(e_{\varphi_{1}}\right)[\mathrm{rad}]$ & 0.0267 & 0.0182 & 0.0060 & 0.0019 \\
$\max \operatorname{std}\left(e_{\varphi_{2}}\right)[\mathrm{rad}]$ & 0.3427 & 0.2671 & 0.0451 & 0.0071 \\
$\max \operatorname{std}\left(v_{1}\right)[\mathrm{m} / \mathrm{s}]$ & 2.4941 & 0.4240 & 0.1868 & 15.4029 \\
$\max \operatorname{std}\left(v_{2}\right)[\mathrm{rad} / \mathrm{s}]$ & 21.0354 & 4.2751 & 0.6539 & 0.1144 \\
$\left.\operatorname{std}\left(v_{1}\right)\right|_{\alpha^{*}}[\mathrm{~m} / \mathrm{s}]$ & 0.5754 & 0.2785 & 0.0914 & 0.1816 \\
$\left.\operatorname{std}\left(v_{2}\right)\right|_{\alpha^{*}}[\mathrm{rad} / \mathrm{s}]$ & 2.1066 & 0.1424 & 0.0024 & 0.0006 \\
\hline
\end{tabular}

4 quite high maximum variance of signal $v_{1}$ is recorded when classic TF is used. This phenomenon follows from the significant angular errors which can be met for the given $\varepsilon$. Then input transformation with matrices $U$ and $T$ defined by Eqs. 14 and 1, respectively, become worse definite and the resultant open loop gain is increased. Again, this is a drawback of local domain of the coordinate transformation $\Phi$. This effect can be confirmed by comparing deviation of input signals presented in Tables 3, 4, 6 and 7 .

The next observation comes from comparison of results obtained for $N=1$ and $N=2$. It is clear that for the higher dimensional system difficulty of control increases while the robustness decreases. Hence, no results for $\varepsilon=0.25$ is presented for
$N=2$-in such a case the sensitivity is enormously high.

Another issue can be discussed comparing minimum standard deviation of $v_{2}$ denoted by $\left.\operatorname{std}\left(v_{2}\right)\right|_{\alpha^{*}}$ and the corresponding value $\left.\operatorname{std}\left(v_{1}\right)\right|_{\alpha^{*}}$ with maximum deviations $\max \operatorname{std}\left(v_{1}\right)$ and $\max$ $\operatorname{std}\left(v_{2}\right)$. Consequently, it follows that the sensitivity of the closed-loop control system is dependent on variable $\alpha$. This property can be quite easily explained evoking dependence of the Frobenius of matrix $P$ on $\alpha$.

Robustness to the Noise-Tracking of Admissible Trajectory Case The last considered simulations are devoted to trajectory tracking. For simplicity it
Table 6 Results of simulations for $N=2$-generalized TF, $\varphi_{r}=0$

\begin{tabular}{lrrll}
\hline Measure & $\varepsilon=0.5$ & $\varepsilon=1$ & $\varepsilon=2$ & $\varepsilon=4$ \\
\hline $\max \operatorname{mean}\left(e_{x}\right)[\mathrm{m}]$ & 0.2277 & 0.0694 & 0.0089 & 0.0012 \\
$\max \operatorname{mean}\left(e_{y}\right)[\mathrm{m}]$ & 0.0009 & 0.0001 & $1 \cdot 10^{-7}$ & $1.4 \cdot 10^{-10}$ \\
$\max \operatorname{mean}\left(e_{\theta}\right)[\mathrm{rad}]$ & 0.0099 & 0.0027 & $1 \cdot 10^{-5}$ & $5.9 \cdot 10^{-8}$ \\
$\max \operatorname{mean}\left(e_{\varphi_{1}}\right)[\mathrm{rad}]$ & 0.0870 & 0.0505 & 0.0019 & $7.6 \cdot 10^{-5}$ \\
$\max \operatorname{mean}\left(e_{\varphi_{2}}\right)[\mathrm{rad}]$ & 0.5265 & 0.5998 & 0.1916 & 0.0291 \\
$\max \operatorname{std}\left(e_{x}\right)[\mathrm{m}]$ & 0.0918 & 0.0493 & 0.0126 & 0.0016 \\
$\max \operatorname{std}\left(e_{y}\right)[\mathrm{m}]$ & 0.0010 & 0.0002 & $2 \cdot 10^{-7}$ & $1.5 \cdot 10^{-10}$ \\
$\max \operatorname{std}\left(e_{\theta}\right)[\mathrm{rad}]$ & 0.0087 & 0.0046 & $2 \cdot 10^{-5}$ & $1 \cdot 10^{-7}$ \\
$\max \operatorname{std}\left(e_{\varphi_{1}}\right)[\mathrm{rad}]$ & 0.0421 & 0.0805 & 0.0022 & $4.5 \cdot 10^{-5}$ \\
$\max \operatorname{std}\left(e_{\varphi_{2}}\right)[\mathrm{rad}]$ & 0.3072 & 0.5331 & 0.2467 & 0.0425 \\
$\max \operatorname{std}\left(v_{1}\right)[\mathrm{m} / \mathrm{s}]$ & 6.6034 & 1.9309 & 0.1992 & 0.0324 \\
$\max \operatorname{std}\left(v_{2}\right)[\mathrm{rad} / \mathrm{s}]$ & 86.8814 & 22.7254 & 4.6381 & 0.7488 \\
$\left.\operatorname{std}\left(v_{1}\right)\right|_{\alpha^{*}}[\mathrm{~m} / \mathrm{s}]$ & 2.2640 & 0.3102 & 0.0159 & 0.0139 \\
$\left.\operatorname{std}\left(v_{2}\right)\right|_{\alpha^{*}}[\mathrm{rad} / \mathrm{s}]$ & 22.8461 & 1.6705 & 0.0829 & 0.0033 \\
\hline
\end{tabular}


Table 7 Results of simulations for $N=2$-generalized TF, $\varphi_{r}=\left[\frac{\pi}{3}-\frac{\pi}{3}\right]^{\top}$

\begin{tabular}{lrlll}
\hline Measure & $\varepsilon=0.5$ & $\varepsilon=1$ & $\varepsilon=2$ & $\varepsilon=4$ \\
\hline $\max \operatorname{mean}\left(e_{x}\right)[\mathrm{m}]$ & 0.1979 & 0.0945 & 0.0090 & 0.0015 \\
$\max \operatorname{mean}\left(e_{y}\right)[\mathrm{m}]$ & 0.0209 & 0.0049 & 0.0001 & $3 \cdot 10^{-6}$ \\
$\max \operatorname{mean}\left(e_{\theta}\right)[\mathrm{rad}]$ & 0.1823 & 0.0913 & 0.0093 & 0.0016 \\
$\max \operatorname{mean}\left(e_{\varphi_{1}}\right)[\mathrm{rad}]$ & 0.1794 & 0.0815 & 0.0054 & 0.0007 \\
$\max \operatorname{mean}\left(e_{\varphi_{2}}\right)[\mathrm{rad}]$ & 0.6474 & 0.4369 & 0.0633 & 0.0096 \\
$\max \operatorname{std}\left(e_{x}\right)[\mathrm{m}]$ & 0.0905 & 0.0382 & 0.0124 & 0.0017 \\
$\max \operatorname{std}\left(e_{y}\right)[\mathrm{m}]$ & 0.0136 & 0.0034 & 0.0002 & $3 \cdot 10^{-6}$ \\
$\max \operatorname{std}\left(e_{\theta}\right)[\mathrm{rad}]$ & 0.1036 & 0.0401 & 0.0128 & 0.0018 \\
$\max \operatorname{std}\left(e_{\varphi_{1}}\right)[\mathrm{rad}]$ & 0.0805 & 0.0616 & 0.0073 & 0.0009 \\
$\max \operatorname{std}\left(e_{\varphi_{2}}\right)[\mathrm{rad}]$ & 0.2757 & 0.3472 & 0.0788 & 0.0124 \\
$\max \operatorname{std}\left(v_{1}\right)[\mathrm{m} / \mathrm{s}]$ & 10.5566 & 2.2942 & 0.2966 & 0.0493 \\
$\max \operatorname{std}\left(v_{2}\right)[\mathrm{rad} / \mathrm{s}]$ & 36.0196 & 9.7724 & 1.3524 & 0.2073 \\
$\left.\operatorname{std}\left(v_{1}\right)\right|_{\alpha^{*}}[\mathrm{~m} / \mathrm{s}]$ & 4.6013 & 0.4440 & 0.0495 & 0.0361 \\
$\left.\operatorname{std}\left(v_{2}\right)\right|_{\alpha^{*}}[\mathrm{rad} / \mathrm{s}]$ & 10.6654 & 0.4307 & 0.0255 & 0.0079 \\
\hline
\end{tabular}

is assumed that the reference trajectory is defined by integration of the reference model with initial condition $q_{r}(0)=0$ and inputs given by

$$
\begin{aligned}
& v_{r 1}(t)= \begin{cases}0.3 & \text { if } t<t_{s} \\
0.3 \exp \left(t_{s}-t\right) & \text { if } t \geq t_{s}\end{cases} \\
& v_{r 2}(t)= \begin{cases}\cos (0.5 t) & \text { if } t<t_{s} \\
\cos (0.5 t) \exp \left(t_{s}-t\right) & \text { if } t \geq t_{s}\end{cases}
\end{aligned}
$$

where $t_{s}=30 \mathrm{~s}$. It is worth to note that the reference trajectory gives possibility to consider tracking and regulation problems (for $t>t_{s}$ the evolution of the reference trajectory is gradually frozen).

The controller using generalized TFs was implemented in two versions. The first one is based on Proposition 1 while the second one incorporates suboptimal strategy given by Proposition 2. The first controller is the same
Fig. 8 Results of trajectory tracking using classic feedback for $N=1$, generalized $\mathrm{TF}$ with $\varepsilon=4$

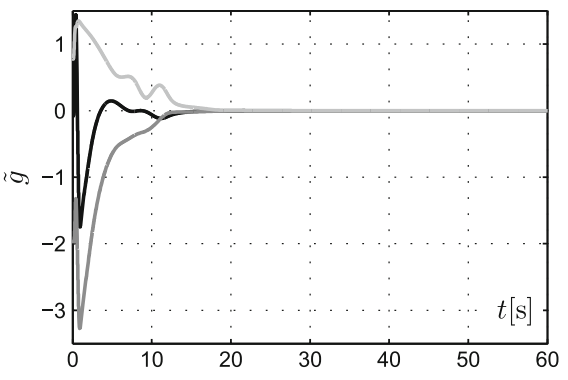

(a) Errors: $\tilde{x} \square, \tilde{y} \square[\mathrm{m}], \tilde{\theta} \square[\mathrm{rad}]$

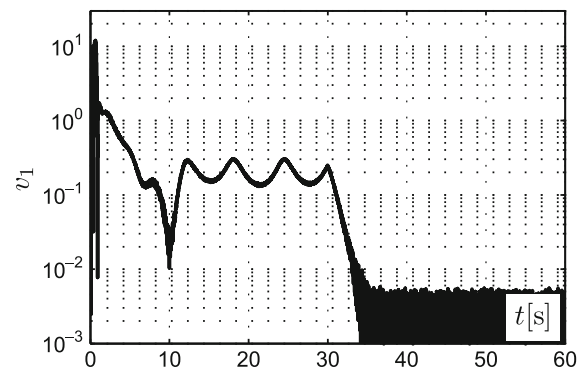

(c) Control input $v_{1}[\mathrm{~m} / \mathrm{s}]$

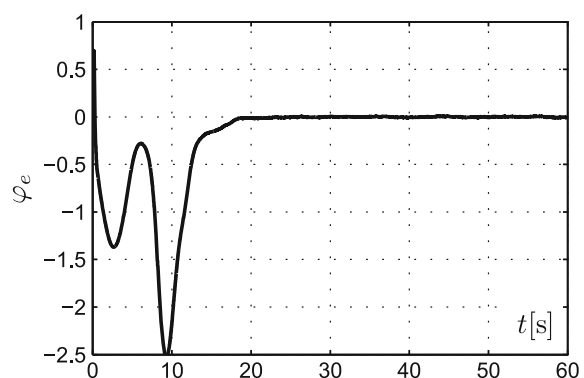

(b) Errors: $\varphi_{e 1} \boldsymbol{\square}[\mathrm{rad}]$

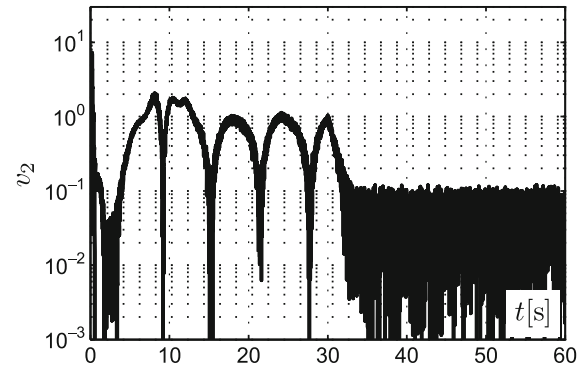

(d) Control input $v_{2}[\mathrm{rad} / \mathrm{s}]$ 
Fig. 9 Results of trajectory tracking using optimized feedback for $N=1$, generalized TF with $\varepsilon=4$

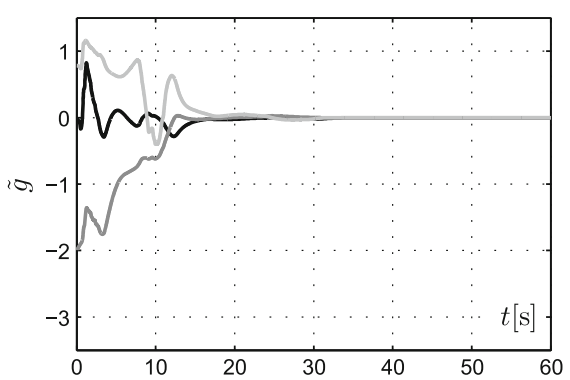

(a) Errors: $\tilde{x} \square, \tilde{y} \square[\mathrm{m}], \tilde{\theta} \square[\mathrm{rad}]$

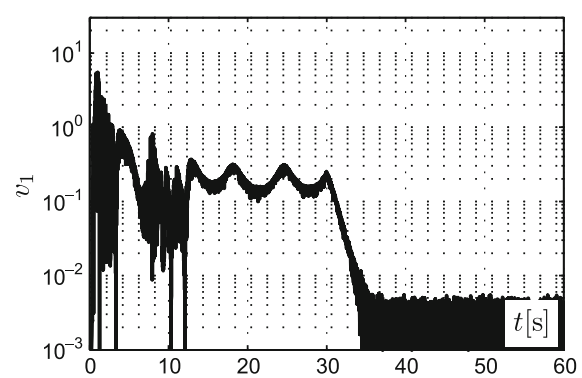

(c) Control input $v_{1}[\mathrm{~m} / \mathrm{s}]$

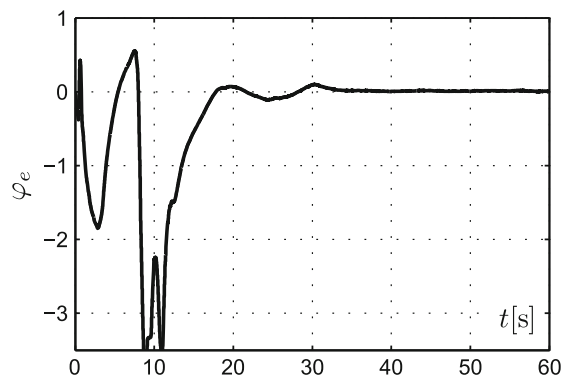

(b) Errors: $\varphi_{e 1} \mathbf{\square}[\mathrm{rad}]$

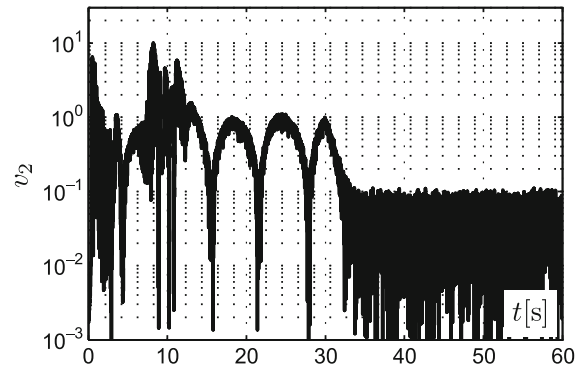

(d) Control input $v_{2}[\mathrm{rad} / \mathrm{s}]$ as in simulations described above. The parameters of the second controller were chosen as follows: $W_{1}=\operatorname{diag}\{10,1,0.01,0.01\}(N=1)$,
$W_{1}=\operatorname{diag}\{10,1,0.01,0.01,0.01\} \quad(N=2), W_{2}=$ $-K=I_{(N+3) \times(N+3)}$ and $\lambda_{s}=\lambda_{d}=10$. The initial configuration error is $q_{e}(0)=\left[\begin{array}{lll}0 & -2 \pi / 4 & 0\end{array}\right]^{\top}$
Fig. 10 Results of trajectory tracking using classic feedback for $N=2$, generalized $\mathrm{TF}$ with $\varepsilon=4$

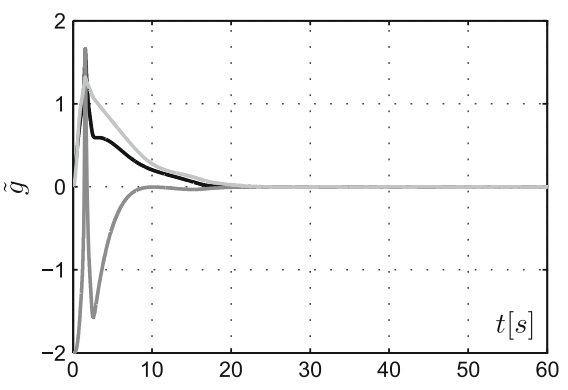

(a) Errors: $\tilde{x} \square, \tilde{y} \square[\mathrm{m}], \tilde{\theta} \square[\mathrm{rad}]$

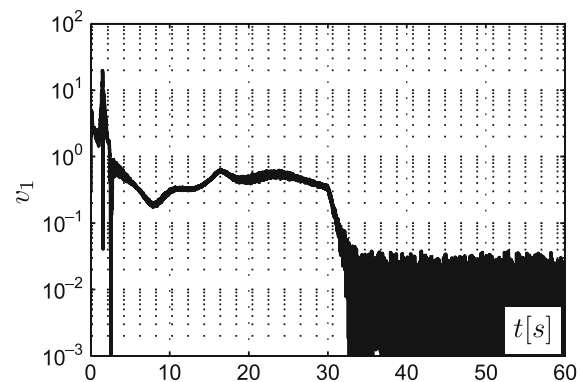

(c) Control input $v_{1}[\mathrm{~m} / \mathrm{s}]$

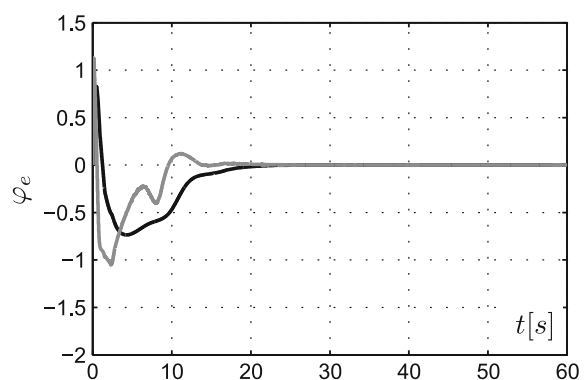

(b) Errors: $\varphi_{e 1} \boldsymbol{\square}[\mathrm{rad}]$

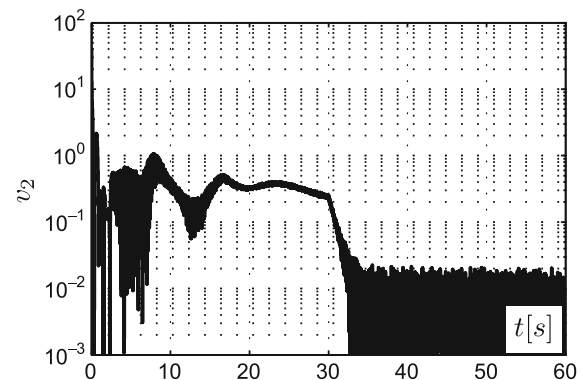

(d) Control input $v_{2}[\mathrm{rad} / \mathrm{s}]$ 
Fig. 11 Results of trajectory tracking using optimized feedback for $N=2$, generalized TF with $\varepsilon=4$

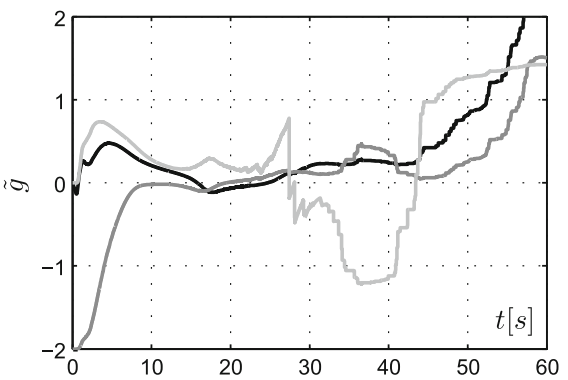

(a) Errors: $\tilde{x} \mathbf{\square}, \tilde{y} \square[\mathrm{m}], \tilde{\theta} \square[\mathrm{rad}]$

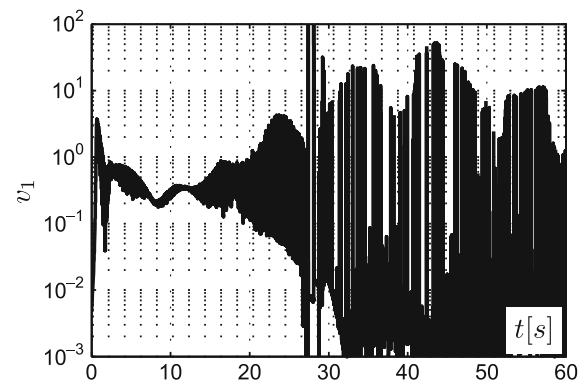

(c) Control input $v_{1}[\mathrm{~m} / \mathrm{s}]$

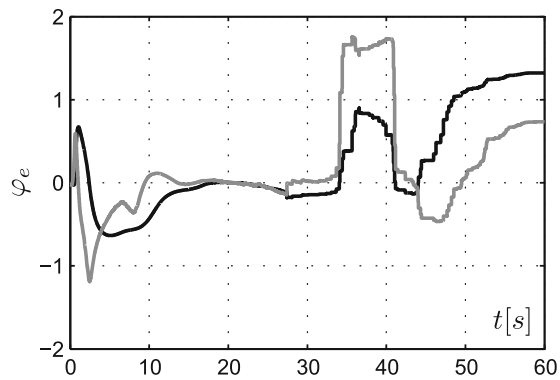

(b) Errors: $\varphi_{e 1} \mathbf{\square}[\mathrm{rad}]$

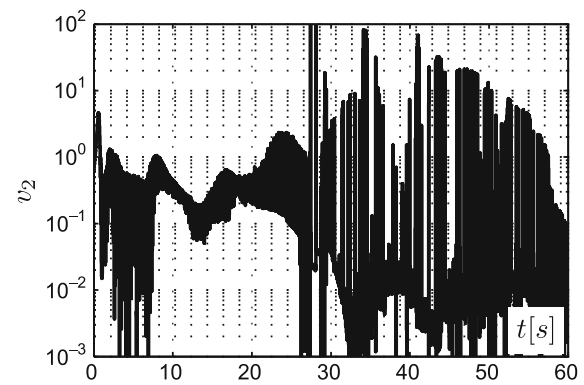

(d) Control input $v_{2}[\mathrm{rad} / \mathrm{s}]$

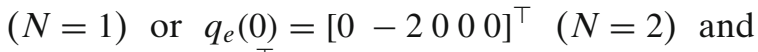
$\alpha_{r}=-\left[\begin{array}{lll}\frac{\pi}{2} & \ldots & \frac{\pi}{2}\end{array}\right]^{\top}$. The results of simulations are presented in Figs. 8, 9, 10 and 11.

It can be concluded that during the transient states the vehicle is moving quite rapidly (no input saturation is taken into account) and magnitudes of the position errors increase significantly. Moreover, it is observed that such a behavior appears when relatively high initial angular errors are present. Referring to the obtained results it is confirmed that the suboptimal control feedback gives possibility to limit extensive motion (in particular magnitude of $\tilde{y}$ is reduced), however the improvement may not be very clear.

The main disadvantage of the suboptimal control solution considered here is the weakness of robustness to the noise. In spite of using quite high value of $\lambda_{s}$ and $\lambda_{d}$ the sensitivity to the measurement noise is significant. Comparing the results obtained for the classic and suboptimal control feedbacks one can notice that for the second solution deviation of the control inputs during transient state is higher. Moreover, the increase of sensitivity with respect to suboptimal controller leads to increase the tracking error. Comparing Figs. 10 and 11 one can observe that at the end of simulation the configuration errors are enormously high for the suboptimal control scheme. Further reduction of the error is not possible by simple decreasing $\varepsilon$ because of the robustness issue.

\section{Conclusions}

In this paper the controller using transverse functions applied for the vehicles with trailers is examined thoroughly. Theoretical considerations are devoted to control design and parametrization of the controller and transverse functions associated with it. It is studied how the transverse function scaling based on dilation affects resultant openloop gain of the controller. Then the optimal method of selection of transverse function parameters using Monte-Carlo approach is considered. In order to make more detailed analysis the controller designed for the vehicle with one and two trailers is taken into account. The simulations results illustrate tracking error bound and robustness of the control system to the measurement noise with respect to transverse function scaling. 
The detailed results allows one to conclude that the implementation of the considered control scheme is quite demanding. Taking into account the controller tuning most care should be devoted to ensuring robustness to the measurement noise. In particular this is difficult when the classic transverse function is used. Then in order to guarantee suitable level of robustness the parameter $\varepsilon$ should be chosen high enough which may deteriorate control accuracy. Recalling the method of gradual scaling based on prediction or adaptation of parameter $\varepsilon$ described in [21] one can state that such an approach is very difficult to apply. It seems that the better solution is to use the generalized transverse function which give possibility to reduce its norm without affecting value of parameter $\varepsilon$. However, it should be mentioned that this method is quite convenient with respect to tracking of some kind of admissible trajectories. Otherwise one can change $\alpha_{r}$ in order to follow current value of $\alpha$. To be more precisely when $\varepsilon$ is made relatively high in order to reduce tracking error $\alpha_{r}$ should stay in some neighborhood of $\alpha$ with small radius. This seems to be a hint for the controller tuning, however implementation of this idea may be a challenge-cf. [12].

The important issue is related to the robustness dependency on $\alpha$ which evolution is governed by the control law. It turns out that in some range of $\alpha$ resultant gain of the open loop increases and the sensitivity of the controller rises. In order to overcome this difficulty one can consider gain scheduling and further modification of a transverse function. This problem is critical and it is not solved so far.

Another problem is attenuation of oscillatory behavior during transient states (when the motion with many maneuvers is not necessary for realization of the task) along with limitation of excessive motions. Theoretically the suboptimal control solution examined in the paper can be used to solve this problem. However, it can hardly be used in practice as a result of robustness requirement.

Summarizing it can be stated that implementation of the considered controller in real application it not an easy task. First, localization system should be very precise. In order to ensure this requirement one can take advantage of observers along with data fusion algorithms. Secondly, limi- tation of unnecessary maneuvers during transient states is quite difficult. This issue can be overcome by planning reference trajectory such that initial configuration error is made relatively small. It it worth to note that in the considered case the difficulty of control problem arises when number of trailers increases, namely demand of computing power and sensitivity becomes an issue for $N \geq 3$.

Concluding, there are still many unsolved aspects which could be taken into account considering the control approach based on transverse functions. In particular one can point out searching for alternative design of transverse function which satisfies theoretical requirements and provide better properties taking into account the real applications.

Open Access This article is distributed under the terms of the Creative Commons Attribution License which permits any use, distribution, and reproduction in any medium, provided the original author(s) and the source are credited.

\section{Appendix}

Dilation of the Transverse Function Consider function scaling using dilation operator, namely

$f^{\varepsilon}=D(\varepsilon) f$

where $f$ is a tranverse function satisfying the transversality condition and $D(\varepsilon)$ is the dilation operator given in the matrix notation. Next, we investigate the transversality condition with respect to function $f^{\varepsilon}$ and define

$$
H=\left[X_{1}^{c}\left(f^{\varepsilon}\right) X_{2}^{c}\left(f^{\varepsilon}\right)-\frac{\partial f^{\varepsilon}}{\partial \alpha}\right] .
$$

Taking into account that $X_{1}^{c}$ and $X_{2}^{c}$ are homogeneous vector field of degree -1 and using that $\frac{\partial f^{\varepsilon}}{\partial \alpha}=D(\varepsilon) \frac{\partial f}{\partial \alpha}$ gives

$$
\begin{aligned}
H & =\left[\varepsilon^{-1} D(\varepsilon) X_{1}^{c}(f) \mid \varepsilon^{-1} D(\varepsilon) X_{2}^{c}(f)-D(\varepsilon) \frac{\partial f}{\partial \alpha}\right] \\
& =D(\varepsilon)\left[\varepsilon^{-1} X_{1}^{c}(f) \varepsilon^{-1} X_{2}^{c}(f)-\frac{\partial f}{\partial \alpha}\right] .
\end{aligned}
$$

Since $X_{1}^{c}, X_{2}^{c}$ and $\frac{\partial f}{\partial \alpha}$ are linearly independent from assumption it follows that matrix $H$ has full rank and the transversality condition is satisfied. 
Scaling of Transverse Function Parameters Consider function given by Eq. 47 with $f$ defined by Eqs. 21 and 22. Referring to dilation properties one has

$$
\begin{aligned}
D(\varepsilon) f(\alpha):= & D(\varepsilon)^{M-2} f\left(\alpha_{M-2}\right) \star D(\varepsilon)^{M-3} \\
& \times f\left(\alpha_{M-3}\right) \star \ldots \star D(\varepsilon)^{1} f\left(\alpha_{1}\right)
\end{aligned}
$$

and

$$
\begin{aligned}
D(\varepsilon)^{i} f\left(\alpha_{i}\right)=\exp ( & D(\varepsilon) X_{1}^{c}\left({ }^{i} f\right) \beta_{i, 1} \sin \alpha_{i} \\
& \left.\left.+D(\varepsilon) X_{i+1}^{c}{ }^{i} f\right) \beta_{i, 2} \cos \alpha_{i}\right) .
\end{aligned}
$$

Next, taking into account that $D(\varepsilon) X_{j}^{c}\left({ }^{i} f\right)=$ $\varepsilon^{-d} X_{j}^{c}\left(D(\varepsilon)^{i} f\right)$, where $d$ is homogeneity degree of vector field $X_{j}^{c}$, and defining ${ }^{i} f^{\varepsilon}:=D(\varepsilon)^{i} f\left(\alpha_{i}\right)$ one obtains

${ }^{i} f^{\varepsilon}=\exp \left(\varepsilon X_{1}^{c}\left({ }^{i} f^{\varepsilon}\right) \beta_{i, 1} \sin \alpha_{i}+\varepsilon^{i} X_{i+1}^{c}\left({ }^{i} f^{\varepsilon}\right) \beta_{i, 2} \cos \alpha_{i}\right)$.

As a result the following new set of parameters can be established

$\beta_{i, 1}^{\varepsilon}:=\varepsilon \beta_{i, 1}, \quad \beta_{i, 2}^{\varepsilon}:=\varepsilon^{i} \beta_{i, 2}$.

\section{References}

1. Artus, G., Morin, P., Samson, C.: Tracking of an omnidirectional target with a unicycle-like robot: control design and experimental results. Research Report INRIA 4849, INRIA (2003)

2. de Wit, C.C., Sørdalen, O.J.: Exponential stabilization of mobile robots with nonholonomic constraints. IEEE Trans. Autom. Control 37, 1791-1797 (1992)

3. Dulęba, I., Sąsiadek, J.: Nonholonomic motion planning based on Newton algorithm with energy optimization. IEEE Trans. Control Syst. Technol. 11(3), 355363 (2003)

4. Dutkiewicz, P., Kiełczewski, M., Kozłowski, K., Pazderski, D.: Vision localization system for mobile robot with velocities and acceleration estimator. Bull. Pol. Ac.: Tech. 58(1), 355-363 (2010)

5. Fliess, M., Levine, J., Martin, P., Rouchon, P.: Flatness and defect of non-linear systems: introductory theory and examples. Int. J. Control. 61(6), 1327-1361 (1995)

6. Jiang, Z.P.: Robust exponential regulation of nonholonomic systems with uncertainties. Automatica 36, 189209 (2000)

7. Lafferriere, G., Sussmann, H.J.: A differential geometric approach to motion planning. In: Nonholonomic Motion Planning, pp. 235-270. Kluwer (1993)

8. Laumond, J.P., Sekhavat, S., Lamiraux, F.: Guidelines in nonholonomic motion planning for mobile robots.
In: Lecture Notes in Control and Information Sciences, Robot Motion Planning and Control, vol. 229, pp. 1-54. Springer-Verlag, Berlin (1998)

9. Lizárraga, D., Morin, P., Samson, C.: Non-robustness of continuous homogeneous stabilizers for affine control systems. In: Proc. of Conference on Decision and Control, pp. 855-860. Phoenix, USA (1999)

10. Michałek, M., Kozłowski, K.: Vector-Field-Orientation feedback control method for a differentially driven vehicle. IEEE Trans. Control Syst. Technol. 18(1), 45-65 (2010)

11. Morin, P., Samson, C.: A characterization of the Lie Algebra rank condition by transverse periodic functions. SIAM J. Control Optim. 40, 1227-1249 (2001)

12. Morin, P., Samson, C.: Practical and asymptotic stabilization of the 3-D chained system by the transverse function approach. In: Proceedings of 42nd IEEE Conference on Decision and Control, vol. 5, pp. 4700-4704 (2003)

13. Morin, P., Samson, C.: Practical stabilization of driftless systems on Lie groups: the transverse function approach. IEEE Trans. Autom. Control 48(9), 1496-1508 (2003)

14. Morin, P., Samson, C.: Motion control of wheeled mobile robots. In: Springer Handbook of Robotics, pp. 799-826. Springer, Berlin Heidelberg (2008)

15. Morin, P., Samson, C.: Trajectory tracking for nonholonomic systems. Theoretical background and applications. Rapport de recherche RR-6464, INRIA (2008). URL: http://hal.inria.fr/inria-00260694

16. Morin, P., Samson, C.: Transverse function control of a class of non-invariant driftless systems, application to vehicles with trailers. In: Proceedings of 47th IEEE Conference on Decision and Control, pp. 4312-4319 (2008)

17. Morin, P., Samson, C.: Control of nonholonomic mobile robots based on the transverse function approach. IEEE Trans. Robot. 25(5), 1058-1073 (2009)

18. Murray, R., Sastry, S.S.: Nonholonomic motion planning: steering using sinusoids. IEEE Trans. Autom. Control 38, 700-716 (1993)

19. Pazderski, D., Kozłowski, K.: Practical stabilization of two-wheel mobile robot with velocity limitations using time-varying control law. In: Proceedings of the 5th International Workshop on Robot Motion and Control, pp. 205-212 (2005)

20. Pazderski, D., Kozłowski, K.: Trajectory tracking of underactuated skid-steering robot. In: Proceedings of the American Control Conference, pp. 3506-3511 (2008)

21. Pazderski, D., Kozłowski, K., Waśkowicz, D.: Control of a unicycle-like robot with trailers using transverse function approach. Bull. Pol. Ac.: Tech. 60(3), 537-546 (2012)

22. Pazderski, D., Krysiak, B., Kozłowski, K.: A comparison study of discontinuous control algorithms for threelink nonholonomic manipulator. In: Lecture Notes in Control and Information Sciences, pp. 35-44. SpringerVerlag, Berlin Heidenberg (2012)

23. Pomet, J.B.: Explicit design of time varying stabilization control laws for a class of controllable systems without drifts. Syst. Control Lett. 18(2), 147-158 (1992) 
24. Pomet, J.B., Thuilot, B., Bastin, G., Campion, G.: A hybrid strategy for the feedback stabilization of nonholonomic mobile robots. In: Proceedings of the 1992 IEEE International Conference on Robotics and Automation, pp. 129-134. Nice, France (1992)

25. Prieur, C., Astolfi, A.: Robust stabilization of chained systems via hybrid control. IEEE Trans. Autom. Control 48(10), 1768-1772 (2003)
26. Samson, C.: Velocity and torque feedback control of a nonholonomic cart. In: de Wit, C.C. (ed.) Advanced Robot Control, pp. 125-151. Birkhauser, Boston, MA (1991)

27. Sørdalen, O.J., de Wit, C.C.: Exponential control law for a mobile robot: extention to path following. IEEE Trans. Robot. Autom. 9(6), 837-842 (1993) 